\title{
LE TRE ONDATE DELL'ESTREMISMO DI DESTRA IN GERMANIA OCCIDENTALE
}

\author{
di Ekkart Zimmermann e Thomas Saalfeld
}

\section{Introduzione}

«Non sarà un secondo Hitler a sfidare lo stato di Bonn, nessun imitatore che possa accattivarsi la nostra attenzione. Sarà un originale senza precedenti, una personalità adeguata alle caratteristiche e ai bisogni del suo tempo. Le rassomiglianze risulteranno evidenti al massimo agli esperti, ma non all'elettorato di massa. La seduzione si avvicina alle sue vittime con immaginazione e fantasia, non secondo stereotipi e forme riconoscibili» (Neubauer 1985, 135, traduzione dal tedesco all'inglese degli Autori).

Anche se oggi le condizioni politiche non corrispondono assolutamente a ciò che è suggerito in questa citazione di un ex leader politico dei Republikaner tedeschi (occidentali), è bene che l'opinione pubblica sia avvertita. I successi elettorali dei partiti di estrema destra nel 1989 e nel 1991-1992, così come il preoccupante numero di attacchi violenti di neonazisti $\mathrm{e}$ skinheads contro immigrati, rifugiati in cerca di asilo, istituzioni e cimiteri ebraici, monumenti antifascisti, senza casa e altre minoranze, possono essere visti come esempi lampanti di una rinascita dell'estremismo di destra in Germania. Una solida analisi di questo fenomeno è più rilevante delle profezie interessate dei leader dell'estrema destra. Che l'estremismo di destra possa rappresentare una sfida alla democrazia liberale è una delle lezioni della caduta della Repubblica di Weimar. Comprendere le condizioni e ciò che accompagna il suo successo, dunque, può contribuire al contenimento di questo pericolo.

Questo articolo si divide in tre parti. Dopo aver caratterizzato gli aspetti principali dell'estremismo di destra, si procederà a un'analisi longitudinale delle tre ondate più forti dell'estremismo di destra nella Germania Occidentale dalla seconda guerra 
mondiale ad oggi. In questo modo continuità e cambiamenti dovrebbero risultare evidenti. Si farà uso della ricca mole di dati empirici disponibili per tracciare un modello teorico semplice e parsimonioso in grado di rendere conto dell'estremismo di destra in Germania Occidentale (e forse anche in altri paesi occidentali). Il fuoco di questo articolo è puntato sulla Germania Ovest. Si offrirà un breve resoconto degli eventi successivi all'unificazione, ma lo sforzo teorico si concentrerà sulla «vecchia» Repubblica Federale, perché gli studiosi, nel mondo accademico, stanno appena cominciando a prendere le misure della complessa situazione della Germania Est.

\section{Definire l'estremismo di destra}

Si definisce qui come «estremismo di destra» quell'insieme di tentativi, sviluppati da sette, movimenti e partiti politici, di influenzare lo sviluppo della società in modo che siano possibilmente abolite le regole democratiche del gioco politico e che sia, invece, introdotta una ideologia politica e sociale caratterizzata da ultranazionalismo e etnocentrismo (espresso, per esempio, contro i lavoratori stranieri e i profughi in cerca d'asilo), antiamericanismo, anticomunismo, antiparlamentarismo, antipluralismo, antiegualitarismo, militarismo, idee del tipo «leggee-ordine», domanda di leadership politica e/o di un esecutivo forte e carismatico, pessimismo culturale, nozioni corporative in politica $\mathrm{e}$, in una certa misura, in economia. A livello individuale una simile ideologia corrisponde spesso a sistemi di credenze rigidi, stereotipi amico/nemico, attitudine al sospetto, fanatismo e attivismo. Ciò che soggiace a questi obiettivi è lo sforzo di correggere radicalmente sviluppi sociali ed economici che sono percepiti come sfavorevoli sia per l'individuo che per la società nel suo insieme. Nell'invocare tali obiettivi, spesso vengono anche invocati valori e forme di organizzazione di un periodo storico precedente. Nel presente articolo ci si riferirà prevalentemente ai partiti, tralasciando l'analisi delle sette e dei movimenti politici. Con pochi aggiustamenti, tuttavia, analoghe definizioni si potrebbero applicare anche a questi altri fenomeni associativi di destra.

La classificazione di un partito come partito di estrema destra viene compiuta sulla base degli slogan, delle piattaforme elettorali, dei discorsi pubblici e delle interviste di leader e alti 
dirigenti del partito, dei numerosi materiali tratti da indagini in profondità su simpatizzanti del partito, e, anche, del consenso della letteratura specializzata sul tema. Ogni classificazione di questo tipo non può non contenere elementi arbitrari; tuttavia, non c'è altra scelta. Un'analisi formale del contenuto delle piattaforme di partito o un'analisi letterale dei discorsi tenuti in campagna elettorale potrebbero essere fuorvianti, dato che spesso i partiti di estrema destra mettono in sintonia la loro retorica con i requisiti democratici. Per arrivare a una simile classificazione, dunque, conviene usare dati di varia fonte. I risultati ottenuti collocando un partito all'estrema destra in base a quanto fanno sia gli aderenti che gli oppositori di quel partito (Niedermayer 1990, 570-571, che usa dati tratti dall'Eurobarometro) giustificano ulteriormente la nostra procedura. In un certo senso, quest'ultimo sistema è il più forte indicatore di atteggiamenti, giacché rivela qualcosa di più della retorica, spesso pseudo-democratica, dei leader di partito di estrema destra. In passato, del resto, chi ha fatto ricerca su questi fenomeni ha sempre dovuto seguire una strategia analoga di combinazione di elementi definitori bard e soft nella classificazione di un partito di estrema destra. Qualunque altra strategia sottostimerebbe il pericoloso potenziale di simili partiti. Che poi questi stessi partiti possano adattarsi, almeno in parte, ad altre issues e a uno stile di autopresentazione più democratico non altera la loro opposizione di fondo al pluralismo democratico.

\section{I partiti}

$\grave{E}$ impossibile, nelle dimensioni di questo articolo, prendere in esame l'intero spettro dell'estremismo di destra nella Germania occidentale (cfr. Stöss 1989). Perciò, ci concentriamo su: $a$ ) i partiti di estrema destra che hanno ottenuto almeno il cinque per cento dei voti in almeno una tornata elettorale a livello nazionale o di Land $^{1}$ e $b$ ) le organizzazioni non partitiche che non abbiano una base confinata in regioni specifiche. Sei partiti politici soddisfano il nostro criterio del «cinque per cento»: il

${ }^{1}$ Si è scelto il criterio del $5 \%$ perché, secondo la legge elettorale tedesca, un partito si assicura una rappresentanza parlamentare se ha ottenuto almeno il $5 \%$ del voto nazionale, o se almeno tre dei suoi candidati sono riusciti a raggiungere la maggioranza relativa nei loro collegi. 
Partito Socialista del Reich (SRP, 1949-1952), il Partito del Reich Tedesco (DRP, 1950-1965), la Comunità Tedesca (DG, 1949-1965), il Partito Nazionale Democratico Tedesco (NPD, 1964-), il Partito dei Republikaner (1983-) e l'Unione del Popolo Tedesco (DVU, 1971-)2. Ci occuperemo di questi partiti prima di rivolgere l'attenzione alle organizzazioni non partitiche. La DVU sarà analizzata insieme alle organizzazioni non partitiche, in quanto non si è impegnata in una competizione elettorale fino al 1987 e ha svolto la maggior parte delle sue attività al di fuori dell'arena elettorale.

Il Partito Socialista del Reich (SRP) fu fondato nell'ottobre 1949 , in seguito all'abolizione del sistema di autorizzazione dei partiti politici da parte degli Alleati. In termini di ideologia, obiettivi politici e leadership, il partito era un successore della NSDAP. I leader della SRP consideravano l'ultimo gabinetto nazionalsocialista, guidato dall'ammiraglio Dönitz, il solo governo legittimo della Germania e vedevano il sistema di Bonn e il gabinetto Adenauer come una emanazione illegittima delle potenze alleate. «La SRP propagandava una dottrina tipicamente nazista del Reich, intendendolo come una specie di mistica unione di sangue del popolo tedesco. I dirigenti del partito dichiaravano che la Germania avrebbe dovuto diventare una "democrazia fondata sulla leadership" governata da un "Capo $\mathrm{Na}$ zionale" che avrebbe diretto la nazione in qualità di capo dell'esecutivo e con l'assistenza di un parlamento corporativo. La SRP esprimeva apertamente la sua ammirazione per Hitler e il suo regime, sostenendo di voler mantenere i lati positivi del nazismo e portare a compimento la rivoluzione nazionalsocialista» (Fisher 1974, 129-130). Il consenso per la SRP era concentrato nelle zone protestanti della Germania settentrionale. Quasi due terzi dei circa diecimila membri che contava nel 1951 vivevano nella Bassa Sassonia. La sua organizzazione era rigida e gerarchica, in consonanza con il principio della leadership nazionalsocialista. Il suo maggior successo elettorale lo ottenne nel 1951, quando raggiunse l'undici per cento del voto alle elezioni della Dieta statale (Landtag) della Bassa Sassonia, e il 7,7\% a Brema. Sempre nel 1951, il governo di Adenauer ricorse alla Corte Costituzionale Federale per mettere al bando la SRP in

2 I nomi tedeschi sono Sozialistische Reichspartei (SRP), Deutsche Reichspartei (DRP), Deutsche Gemeinschaft (DG), Nationaldemokratische Partei Deutschland (NPD), Die Republikaner (REP) e Deutsche Volksunion (DVU). 
ragione del suo programma antidemocratico e apertamente nazionalsocialista. Nel 1952 il partito venne messo fuori legge. In seguito, la gran parte dei dirigenti della SRP si riversò nel Partito del Reich Tedesco (DRP). I suoi elettori, di contro, finirono per essere recuperati, in larga misura, dai partiti borghesi prosistema (Schmollinger 1986d; Fisher 1974, 129).

Dopo la messa al bando della SRP, il Partito del Reich Tedesco (DRP) rimase il più forte partito di estrema destra degli anni cinquanta e dei primi anni sessanta. La sua esistenza coprì il periodo 1950-1965. Prima del 1952 il partito aveva giocato un ruolo secondario rispetto alla SRP. La maggior parte dei suoi leader erano ex funzionari nazionalsocialisti, e un'ampia percentuale dei suoi membri aveva appartenuto al Partito $\mathrm{Na}$ zionalsocialista. Agli inizi degli anni sessanta, la DRP aveva circa quattromilacinquecento membri; fra questi, approssimativamente, il cinquanta per cento era costituito da ex nazionalsocialisti e il venti per cento da ex membri della SRP3. Il suo sostegno era per lo più limitato a quelle regioni che erano state roccaforti della SRP. Il partito prese parte a tre elezioni federali (1953, 1957, 1961), ma non riuscì mai a raccogliere più dell'1,1\% dei voti totali (1953). I migliori risultati in assoluto della DRP alle elezioni nei Länder furono ottenuti tra il 1955 e il 1959, quando la DRP registrò il 3,8\% dei voti nelle elezioni della Bassa Sassonia (1955) e di Brema (1959), il 3,6\% di nuovo in Bassa Sassonia (1959) e il 5,1\% nella Renania-Palatinato $(1959)^{4}$. Alla sua fondazione (1950), si iscrissero alla DRP cinque membri del Bundestag (1949-1953). Il partito fu rappresentato anche nelle Diete della Bassa Sassonia (1951-1959) e della Renania-Palatinato (1959-1963) (Schmollinger 1986b, 1181; Fisher 1974, 131-132).

Nei loro discorsi programmatici, i leader della DRP si preoccupavano di rispettare formalmente la Legge Fondamentale più di quelli della SRP; tuttavia, per lo più, i loro temi propagandistici si assomigliavano. «I programmi di partito gettavano dubbi e calunnie sulla onestà e il patriottismo dei leader del go-

${ }^{3}$ Le cifre percentuali sono tratte da Fisher $(1974,132)$. Resta poco chiaro fino a che punto gli ex membri della NSDAP e quelli della SRP si sovrappongano.

${ }^{4}$ Cioè, nelle elezioni in cui la DRP non era parte di una coalizione elettorale di destra, come in Baviera (1954) e Brema (1963), dove presentava candidature comuni rispettivamente con il Gesamtdeutscher Block / BHE e il Partito Tedesco (DP), ma non poteva assicurarsi alcun seggio parlamentare per i propri rappresentanti. 
verno, esprimevano la credenza in una comunità nazionale tedesca (Volksgemeinschaft), chiedevano il rilascio di tutti i criminali di guerra tedeschi, invocavano la creazione di un "nuovo Reich" con una vera cultura tedesca, enfatizzavano ciò che c'era di "buono" nel Terzo Reich, domandavano la restaurazione delle frontiere storiche della Germania e insistevano sul ritiro delle truppe straniere» (Fisher 1974, 132). Nel 1964, i leader della DRP promossero e controllarono la fondazione del Partito Nazionale Democratico Tedesco (NPD). In seguito alla formazione della NPD nel 1964, la DRP si dissolse. I suoi membri, i leader e l'organizzazione divennero la spina dorsale della NPD (Schmollinger 1986b).

Mentre la SRP e la DRP ebbero le loro roccaforti in alcune delle regioni protestanti della Germania settentrionale, la Comunità Tedesca (DG) raggiunse una dimensione significativa solo nel Sud, specialmente nel Baden-Württemberg e in Baviera. «La DG in origine cercò di attrarre chi si era impoverito alla fine della guerra, in particolare chi era stato privato della casa dalle forze occupanti, le vittime dei bombardamenti, chi era stato prigioniero di guerra, e chi aveva perso denaro a causa della riforma valutaria» (Fisher 1974, 136). In paragone con il suo principale rivale di estrema destra, la DRP, la DG ha perseguito con più coerenza una politica estera di segno nazionalista-neutralista, chiedendo la fine del «dominio delle internazionali comuniste e capitaliste sul suolo tedesco». Le sue politiche economiche erano meno conservatrici e davano più enfasi ad un «socialismo» piccolo-borghese che implicava la distruzione del potere del capitalismo finanziario e la nazionalizzazione delle industrie-chiave (Fisher 1974, 136). Per questo aspetto, le sue politiche ricordavano i programmi del Partito Nazionalsocialista agli inizi degli anni venti. Il partito riscosse alcuni successi elettorali iniziali in un'alleanza con la Unione degli Espulsi e dei Rifugiati (BHE) in Baden-Württemberg $\mathrm{e}$ in Baviera. «L'alleanza fra questi due movimenti ebbe fine non appena la BHE si schierò a favore della partecipazione al governo e la DG scelse di perseguire una linea di opposizione radicale. [...] Quando la DG cominciò a mettere sotto accusa il regime parlamentare, gli Alleati Occidentali e il governo di Bonn, i leader della $\mathrm{BHE}$ finirono per vedere la DG come un handicap agli sforzi della BHE di ottenere incarichi di governo. Così l'alleanza fu sciolta e, intorno al 1952, l'elettorato profugo della DG si era trasferito quasi per intero 
nella BHE. Da allora in poi, la DG non raccolse più alcun successo elettorale significativo» (Fisher 1974, 136).

Il Partito Nazionale Democratico Tedesco (NPD) venne fondato nel 1964 dalla fusione della DRP con numerosi gruppuscoli di estrema destra. Nella sua fase iniziale nella base del partito si erano riversati in gran numero gli ex nazionalsocialisti (Fisher $1974,143-144)^{5}$. Come la più gran parte dei partiti estremisti di destra, la NPD sottolineava formalmente il suo rispetto per la Legge Fondamentale. I temi fondamentali della sua propaganda, nel periodo di maggior successo, la fine degli anni sessanta, furono «la riabilitazione del nazionalismo e del passato nazionalsocialista; un astio profondo per tutte le cose non tedesche; la denuncia del declino della moralità, dei valori tradizionali e della presunta "messa in liquidazione" del Paese da parte del nemico interno; l'incitamento al ritorno alla disciplina militaristica e autoritaria» (Fisher 1974, 145-146). A questi atteggiamenti si sono aggiunti poi un antiamericanismo, un anticomunismo e un etnocentrismo radicali. Il partito ha come scopo l'instaurazione di una «comunità nazionale» definita su base etnica, basata su regole decisionali corporative invece che competitive, e diretta da un esecutivo forte e non partitico. Chiede inoltre una riduzione significativa degli emigranti e dei profughi con diritto d'asilo. Prima che cominciasse il processo di unificazione tedesca (1990), il partito voleva arrivare alla riunificazione nazionale attraverso quella che era la misura programmatica principale di tutti i partiti di estrema destra, e cioè la neutralità della Germania. La NPD sostiene una «terza via» tra capitalismo e comunismo. Nel suo nuovo programma, che sottolinea la protezione dell'ambiente e il rifiuto degli impianti ad energia nucleare, il partito cerca di modernizzarsi e di sfuggire alla sua precedente immagine neonazista (Paul 1989, 18-19).

Fra il 1966 e il 1968 la NPD superò la barriera del 5\% prevista dalla legge elettorale della Germania Ovest nelle elezioni delle Diete di sette degli otto Länder di quel periodo. Complessivamente, era rappresentata da 61 membri in sette parlamenti statali e si era costruita una solida base organizzativa nella Germania meridionale (Baviera e Baden-Württemberg), dove ai

5 Nel 1966, erano stati membri della NSDAP o di qualche movimento radicale di destra postbellico il $35 \%$ di tutti i membri della NPD, il $46 \%$ dei suoi dirigenti a livello locale e di contea, il $67 \%$ dei suoi funzionari a livello di Land e il $73 \%$ dei suoi funzionari a livello nazionale (Fisher 1974, 143). 
suoi predecessori, la SRP e la DRP, era mancato il successo. Da allora, la base elettorale e organizzativa dell'estremismo di destra tedesco si è spostata dal Nord al Sud della Germania. Comunque, col $4,3 \%$ del totale dei voti, la NPD non riuscì a ottenere una rappresentanza parlamentare nelle elezioni del Bundestag del 1969. Dopodiché, le sue fortune elettorali colarono a picco. Fra il 1972 e il 1990, il suo miglior risultato alle elezioni del Bundestag fu lo 0,6\% (1972 e 1987) (Stöss 1989, 136). Gli insuccessi elettorali fomentarono accese dispute intestine sulla strategia del partito. Mentre la leadership e la maggioranza degli attivisti continuarono a preferire la ricerca di una rappresentanza parlamentare ed eventualmente di una coalizione con uno dei partiti borghesi più radicati, una minoranza di militanti chiedeva una strategia più radicale. Lo sforzo di questi ultimi non ebbe esito. Sicché la maggior parte dei militanti abbandonò il partito all'inizio degli anni settanta; molti di essi, specialmente i membri più giovani, entrarono in gruppi nazionalisti rivoluzionári o neofascisti. Il numero degli iscritti declinò da un massimo di 28.000 nel 1967 e nel 1969 a 6.100 nel 1991 (Schmollinger 1986c; Stöss 1989, 135-191; Schmidt 1968; Bundesministerium des Innern 1992, 130).

Nel 1983 due ex deputati federali della CSU, Franz Handlos e Ekkerard Voigt, e Franz Schönhuber, un giornalista di primo piano che era stato a lungo vicino all'ex leader e primo ministro dello stato federale bavarese Franz Josef Strauss, fondarono il partito dei Republikaner. Handlos e Voigt avevano lasciato la CSU perché in disaccordo con la politica di Strauss nei confronti della Repubblica Democratica Tedesca, che giudicavano troppo conciliante 6 . Dopo alcune dispute interne, il primo leader dei Republikaner, Handlos, lasciò il suo incarico nel 1985 e Schönhuber divenne il suo successore. Questo cambiamento di leadership comportò uno spostamento politico da un orientamento fortemente conservatore, proprio di Handlos e Voigt, a uno più nazionalista. Di conseguenza, il partito dei $R e-$ publikaner risultò più attraente per i membri dei partiti tradizionali dell'estrema destra. Il programma del partito fa da ponte tra il conservatorismo tradizionale, rappresentato dalla destra

6 Strauss aveva organizzato per la Germania Est un grande prestito bancario di un miliardo di marchi tedeschi, che fu rifiutato dagli attivisti conservatori della CSU, in quanto ritenuto una forma inappropriata di stabilizzazione del regime comunista della Germania Est. 
della CDU/CSU, e gli atteggiamenti di estrema destra, articolati dalla NPD e dalla DVU (Backes 1990, 11; Paul 1989, 41-42; sulla DVU, vedi oltre). Fin dalla sua fondazione, il partito dei Republikaner è stato caratterizzato da profonde divisioni interne fra un'ala relativamente moderata che preferisce una strategia conservatrice, ponendo l'accento su temi nazionalistici e di tipo «legge-e-ordine», e coloro i quali vorrebbero una cooperazione più intensa con i partiti tradizionalmente all'estrema destra come la NPD e la DVU.

Il partito dei Republikaner può essere concepito come un partito di protesta radicale e populista di estrema destra (Backes 1990, 13-14; Saalfeld 1993). Esso cerca di presentarsi come un partito pro-sistema, che mette in risalto il rispetto della legge e dell'ordine e che pretende di rappresentare gli interessi della gente comune. Il suo obiettivo programmatico centrale, fino al 1990, era la riunificazione della Germania. La visione apologetica della storia tedesca durante l'era nazista proposta dai Republikaner e le loro aggressive agitazioni contro immigrati, profughi e lavoratori stranieri, giudicati un pericolo per l'identità nazionale della Germania e per l'ordine pubblico, ricalcano le classiche affermazioni dei partiti di estrema destra (Saalfeld 1993). Nelle sue piattaforme programmatiche, il partito dei Republikaner cerca di mobilitare la protesta contro certi mali dello stato e della società, contro i partiti dell'establishment, specialmente contro la CDU/CSU e il governo federale in carica. Contribuiscono inoltre all'impressione circa la sua natura di partito di protesta populista la sua mancanza di una organizzazione in piena regola, le limitate opportunità di partecipazione e il deficit di democrazia intrapartitica (Lepszy 1989, 8-9).

Gli Uffici per la Protezione della Costituzione, a livello federale e di Länder, hanno raccolto prove sufficienti che parti significative della propaganda dei Republikaner hanno contenuti razzisti. Perciò, nel dicembre 1992, i ministri degli Interni della Federazione e dei Länder si sono accordati per porre il partito dei Republikaner sotto sorveglianza in tutto il Paese attraverso gli Uffici per la Protezione della Costituzione. In precedenza, solo la Renania del Nord-Westfalia e Amburgo avevano preso misure simili per controllare le attività dei Republikaner. La decisione sembra essere stata motivata da considerazioni politiche più che da fatti nuovi. Probabilmente, il governo federale e $\mathrm{i}$ governi dei Länder hanno voluto mostrare la loro determinazione a combattere l'estremismo di destra, per scoraggiare gli im- 
piegati pubblici (per esempio, i poliziotti) dall'iscriversi in misura significativa al partito dei Republikaner e per scoraggiare $\mathrm{i}$ politici più di destra dell'Unione dal cooperare in modo più stretto con gli stessi Republikaner ${ }^{7}$.

Nel 1989, anno del loro primo successo elettorale e organizzativo di rilievo, il numero degli iscritti al partito dei Republikaner salì da ottomila (gennaio 1989) a circa venticinquemila unità (gennaio 1990). Benché non vi siano cifre affidabili sull'iscrizione al partito, sembra che questo stesso numero sia sceso attorno alle quindicimila unità nell'autunno del 1990. Tuttavia, per effetto della sua espansione in Germania Est, questa cifra risalì poi a ventimiladuecento nel febbraio 1991 (Veen et al. $1991,21)$. Sotto il profilo organizzativo, il partito è sempre stato più forte in Baviera, Baden-Württemberg e a Berlino Ovest. Buona parte dei suoi dirigenti è reclutata in tre bacini di provenienza: ex attivisti della CDU/CSU, funzionari dei gruppi di pressione degli espulsi ed ex attivisti della NPD e della DVU. Il suo primo successo elettorale risale alle elezioni della Dieta bavarese del 1986, quando raccolse il 3\% del totale dei voti. Nelle elezioni della Dieta di Berlino Ovest del 1989, il partito ottenne il $7,5 \%$, risultato che si ripeté lo stesso anno nella sua prima competizione a livello nazionale, alle elezioni per il Parlamento Europeo (7,1\%). Questa elezione a carattere nazionale, tuttavia, confermò che le sue roccheforti continuavano ad essere dislocate nella Germania meridionale: il partito dei Republikaner raggiunse il $14,6 \%$ dei voti in Baviera e l' $8,7 \%$ nel Baden-Württemberg, mentre i suoi risultati nella Germania settentrionale restavano molto modesti. Nel 1990, declinò con la stessa rapidità con cui era sorto. Perfino nella sua roccaforte, la Baviera, nelle elezioni per la Dieta statale quell'anno il partito non riuscì a superare la soglia del cinque per cento $(4,9 \%)$.

L'unificazione della Germania nel 1990 sembrò interrompere l'ascesa dei Republikaner. Parve infatti svuotare di significato la principale domanda politica sull'agenda del partito. Già prima dell'unificazione, nel 1990 non gli fu consentito di partecipare alle elezioni della Volkskammer e delle istituzioni locali della Germania Est. Le elezioni delle Diete statali dell'Est, l'elezione del Bundestag del 1990 e i sondaggi d'opinione indicarono che il sostegno per i Republikaner stava velocemente sce-

7 Si veda Jochen Bölsche, Das Lied vom Tod, in «Spiegel», n. 52, 21 dicembre 1992, pp. 52-57. 
mando. Il partito presentò sue liste alle elezioni delle Diete statali di quattro dei cinque Länder della Germania Est nell'ottobre 1990 , ma il suo miglior risultato fu soltanto un $1,2 \%$. Non riuscì neppure a conseguire un seggio nelle elezioni tenute quello stesso giorno per la Dieta bavarese, nella regione ove aveva potuto disporre dell'organizzazione e del consenso più forti. Nell'elezione del Bundestag del dicembre 1990 ottenne solo il 2,1\% dei voti complessivi e, dunque, neanche un seggio. Quello stesso giorno il partito perse anche la sua rappresentanza nel parlamento di Berlino, essendosi assicurato non più del $3 \%$ dei voti. Ciononostante, le elezioni per la Dieta statale svoltesi nel 1992 in Baden-Württemberg e le elezioni locali nello stato federale di Hesse del 1993 hanno dimostrato che la riunificazione non ha inflitto un colpo fatale al partito dei Republikaner. In Baden-Württemberg il partito ha ottenuto il 10,9\% dei voti e 15 seggi nella Dieta statale. Nelle elezioni locali di Hesse del marzo 1993 ha raccolto oltre il 7\% dei voti, con punte anche molto superiori al $10 \%$ in alcune aree urbane depresse di Francoforte e Kassel (Parkes 1993). Sondaggi d'opinione recenti indicano che il sostegno al partito si è stabilizzato, su scala nazionale, attorno alla soglia del 5\% (Bickerich 1993, 33).

\section{I movimenti}

In Germania Occidentale l'estremismo organizzato di destra non si è presentato soltanto sotto le spoglie di vari partiti politici; questi ultimi rappresentano solo una parte di un movimento sociale di dimensioni più ampie che inquadra i suoi sostenitori $o$ in attività non partitiche $o$ in gruppi che perseguono una strategia combattiva di azione diretta (violenta). Durante l'occupazione alleata, risultava impossibile agli ex attivisti della NSDAP ricostituire il vecchio partito nazionalsocialista. Alcuni di essi si ritirarono dalla politica, altri si rivolsero ai partiti conservatori nazionalisti autorizzati dagli Alleati, molti altri entrarono in organizzazioni «parapolitiche» come la Hilfsgemeinschaft auf $\mathrm{Ge}$ genseitigkeit der ebemaligen Waffen SS (HIAG) fondata nel 1949, la Deutsche Kulturwerk Europäischen Geistes (DKEG) fondata nel 1950 o i circoli riuniti attorno a giornali periodici come l'eurofascista Nation Europa fondato nel 1951. Queste organizzazioni si mobilitarono contro la «denazificazione», glorificarono i soldati tedeschi che avevano preso parte alla seconda 
guerra mondiale e cercarono di stabilire dei contatti con i movimenti politici conservatori. Inoltre, offrirono aiuto ai leader nazionalsocialisti condannati nei processi di Norimberga e alle loro famiglie e tentarono di costruire una rete di contatti, relazioni personali, idee e strategie. Evitarono di fare apertamente propaganda nazionalsocialista, ma fecero circolare un'ideologia etnocentrista e nazionalista che era imperniata sull'idea del Reich tedesco e sui tentativi di riabilitare i presunti «aspetti positivi» del Terzo Reich. In breve tempo si sviluppò un network di estrema destra caratterizzato da un'aspra opposizione alla democrazia di Bonn e da una mentalità di ghetto basata sui miti della cospirazione e della deprivazione (Jaschke 1989, 515-516; Stöss 1989 129-133). In seguito alla messa al bando della SRP nel 1952 e al calo di capacità di attrazione dei partiti di estrema destra alle elezioni dei primi anni cinquanta, molti attivisti della SRP e della DRP si ritirarono dalla politica di partito e tentarono sempre di più di operare tramite organizzazioni non partitiche come quelle sopra menzionate. Ritirarsi nelle reti subculturali ha funzionato, in genere, da strategia di sopravvivenza dell'estremismo di destra tedesco nei tempi di fallimento elettorale. Inoltre, si cercò di mantenere la continuità delle credenze di estrema destra fra le generazioni successive mettendo in piedi organizzazioni della gioventù quale la Wiking Jugend fondato nel 1952 (Jaschke 1989, 517).

Negli anni settanta, dopo l'insuccesso del tentativo della NPD di insediarsi come fattore permanente nella politica tedesco-occidentale, la «vecchia destra», per motivi biografici ancora strettamente legata al nazionalsocialismo, fu in buona parte assorbita dalla Unione del Popolo Tedesco (DVU, Deutsche Volksunion) di Gerhard Frey, fondata nel 1971. Così come era successo alla fine degli anni cinquanta, i network parapolitici svolsero la funzione di «posizioni di riserva», una volta che i maggiori partiti di estrema destra ebbero fallito elettoralmente. Dal 1980 la DVU ha avuto il più alto numero di iscritti fra tutte le organizzazioni di estrema destra (1991: 24.000; Bundesministerium des Innern 1992, 129). Fino al 1987, la DVU ha operato da organizzazionemadre di un insieme di «comunità d'azione» (Aktionsgemeinschaften), che hanno articolato domande politiche quali: un'amnistia generale per i criminali di guerra nazionalsocialisti, il rimpatrio degli immigrati, la riunificazione della Germania entro i suoi confini del 1937 o la riduzione della influenza straniera nei programmi radiotelevisivi tedeschi. I sostenitori della DVU, nei vari 
«comitati d'azione», hanno cercato di proseguire la strategia delle organizzazioni parapolitiche degli anni cinquanta. Solo dal 1987 la DVU ha cominciato a partecipare alle competizioni elettorali. Mentre in precedenza aveva duramente criticato la NPD, tanto da raccomandare perfino ai suoi sostenitori, in qualche occasione, di votare la CDU/CSU alle elezioni politiche, dal 1987 la DVU ha collaborato con la NPD nella maggior parte delle elezioni nazionali. I leader di entrambi i partiti si sono accordati in modo da sostenersi a vicenda in certe tornate elettorali invece di competere l'uno contro l'altro (Stöss 1989, 184-191). Le pubblicazioni della DVU fanno ampio sfoggio di elementi di revisionismo storico, di diniego della responsabilità tedesca per lo scoppio della seconda guerra mondiale e di aggressive campagne antiprofughi (Paul 1989, 18). Nella sua campagna elettorale del 1987, accusò i politici a capo della CDU/CSU di prendere parte alle «istigazioni» straniere contro il popolo tedesco e di essere inerti riguardo a ciò che la DVU concepisce come la minaccia della immigrazione e della «alienazione» etnica. Nelle elezioni europee del 1989 il partito ha anche cercato di mobilitare i sentimenti nazionalisti e antieuropei; ancora una volta, l'immigrazione fu un tema centrale (Paul 1989, 19-20). Tuttavia, con l'1,6\% dei voti, la DVU-Liste $\mathrm{D}$ venne chiaramente scavalcata dal suo rivale più importante, il partito dei Republikaner. Cionondimeno, nelle elezioni dei Länder del 1991 e del 1992, la DVU è riuscita a superare la soglia del $5 \%$ a Brema e nello Schleswig-Holstein. Con poco più del $6 \%$ dei voti in entrambe le tornate elettorali, ottenne sei seggi in ciascuno dei due parlamenti statali.

Oltre alle sue risorse finanziarie relativamente grandi, la spina dorsale della DVU sono i vari quotidiani, periodici e libri di estrema destra pubblicati da Gerhard Frey ${ }^{8}$. Ogni settimana, complessivamente, le pubblicazioni di Frey hanno una circolazione di oltre centomila copie. Esse sono di importanza cruciale per la conservazione delle tradizioni ideologiche e dei valori dell'estrema destra (Jaschke 1989, 519).

I più giovani radicali di destra che vennero delusi dalla strategia «borghese» della NPD e della DVU furono attratti da gruppi d'azione neonazisti come i Socialisti del Popolo o il Fronte d'Azione dei Nazionalsocialisti (ANS) o da gruppi paramilitari come il Gruppo Hoffmann di Sport Militari (messo al

8 «Deutsche Wochenzeitung», «Gesamtdeutscher Anzeigen» e «Deutsche Nationalzeitung». 
bando nel 1983) ${ }^{9}$. La maggior parte di questi gruppi hanno perseguito una strategia aggressiva consistente nell'uso o nella provocazione della violenza politica, specialmente contro gli immigrati e contro i cittadini e le istituzioni ebraiche. I membri di queste organizzazioni sono in parte reclutati da gruppi giovanili delle classi inferiori, fan clubs di calcio e skinheads. I gruppi d'azione neonazisti hanno ingrossato le fila del Partito dei Lavoratori Liberi $(\mathrm{FAP})^{10}$ e mantengono stretti legami personali con i membri della organizzazione giovanile della NPD, i Giovani Nazionaldemocratici (Jaschke 1989, 519; Stöss 1989, 157. 158).

Nei postumi dell'unificazione tedesca, alcuni gruppi legati alla figura di Michael Kühnen sono stati all'avanguardia dell'attività neonazista, specialmente nei Länder dell'Est. Tra queste organizzazioni si annoverano l'Associazione dei Partigiani del Nuovo Fronte (Gesinnungsgemeinschaft der Neuen Front), il Fronte Nazionalista (Nationalistische Front) e l'Alternativa Tedesca (Deutsche Alternative). Le attività dei gruppi neonazisti occidentali nella Germania Est, soprattutto, e la formazione di nuovi gruppi nei nuovi stati federali hanno portato a un aumento sia nel numero di organizzazioni che in quello di loro affiliati. Nel 1991 l'Ufficio Federale per la Protezione della Costituzione ha contato trenta gruppi (rispetto ai 23 del 1989 e ai 27 del 1990), per un totale di circa 2100 attivisti (1989: 1200; 1990: 1400). Il Fronte Nazionalista e l'Alternativa Tedesca sono stati messi al bando dal Ministero degli Interni nel dicembre del 1992. A svolgere altre attività di estrema destra sono alcuni gruppi disorganizzati o debolmente organizzati che comprendono diverse migliaia di skinbeads (1991: 4200). Gli skinbeads della Germania Est sembrano essere caratterizzati da un livello più alto di politicizzazione e sono influenzati dal pensiero nazionalsocialista più fortemente dei loro omologhi ad Ovest, fra i quali la quota di neonazisti pare essere minore (Bundesministerium des Innern 1992, 72, 91; Roberts 1992, 337).

Oltre alle organizzazioni parapolitiche e ai gruppi di attivisti, c'è un terzo filone, composto principalmente da intellettuali che hanno cercato di seguire la nouvelle droite francese e di sviluppare dei fondamenti teorici moderni dell'estremismo di de-

9 I nomi tedeschi sono Volkssozialisten, Aktionsfront nationaler Sozialiste e Webrsportsgruppe Hoffmann.

10 Il nome tedesco è Freie Arbeiter Partei. 
stra, essendosi resi conto che le ideologie di destra tradizionali non sono state in grado di dare risposta ai problemi politici e sociali di oggi. Questo discorso trova per lo più spazio sulla stampa. Gli autori che lo promuovono sono andati in cerca, con consapevolezza e con un certo successo, dei lettori moderati e conservatori, in modo da gettare intellettualmente un ponte tra l'estremismo di destra e il conservatorismo. Gli schemi interpretativi qui sviluppati si ritrovano nei programmi attuali della NPD e del partito dei Republikaner: neutralità fra (quelli che erano un tempo) i due blocchi, riunificazione intesa come confederazione, terza via tra comunismo sovietico e «imperialismo culturale» americano, identità nazionale e patriottismo come surrogati della vecchia idea imperiale del Reich (Jaschke 1989, 519-520; Feit 1987).

\section{Il voto, gli affiliati, la violenza}

La storia organizzativa ed elettorale dell'estremismo di destra organizzato in Germania Ovest è caratterizzata da diversi alti e bassi ciclici. Questi cicli sono riflessi sia nelle cifre degli affiliati ai partiti di estrema destra (fig. 2), sia nella percentuale cumulativa dei voti ottenuti dai partiti di estrema destra a livello regionale e nazionale, ivi comprese le elezioni europee ${ }^{11}$ (fig. 1).

Ogni colonna in fig. 1 rappresenta, in ordine cronologico, la percentuale cumulata dei voti ottenuti dai partiti di estrema destra in tutte le elezioni per il Bundestag, i Landtag e il Parlamento Europeo fra il 1949 e il 1992. Soltanto in cinque elezioni su 144 questa percentuale cumulativa ha superato il $10 \%$ dei voti totali. In sedici elezioni i partiti di estrema destra hanno oltrepassato la soglia del cinque per cento prevista dalla legge elettorale tedesca. Senza contare le elezioni europee del 1989, alle quali i Republikaner riscossero il 7,1\% dei voti, ciò è successo più a livello di Land che a livello nazionale. I partiti estremisti di destra non sono mai riusciti, né individualmente né in coalizione, a ottenere più del cinque per cento in nessuna delle dodici elezioni per il Bundestag. Solo la NPD si avvicinò a questo margine nelle elezioni del parlamento federale del 1969 $(4,3 \%)$. Un secondo sguardo alla fig. 1 potrà mostrare che le

1 Abbiamo incluso tutti i partiti che, sulla base dei loro programmi, sono stati classificati come «partiti antidemocratici di destra» da Stöss (1986, 242-243). 
Percentuale sul totale dei voti

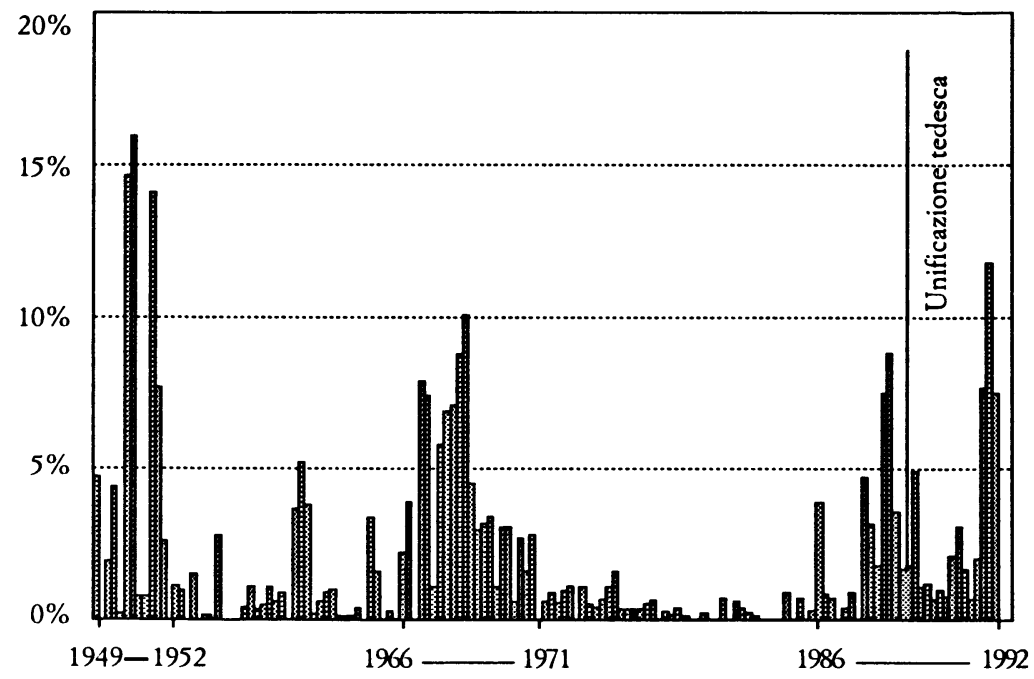

Fig. 1. Percentuali comulative dei voti ottenuti dai partiti di estrema destra in Germania ovest nelle elezioni federali e statali (1949-1992)

elezioni alle quali i partiti di estrema destra hanno avuto un qualche successo relativo non sono distribuite a caso nel tempo. Né è possibile spiegare la variabilità in virtù dei successi ricorrenti in certe roccheforti della destra radicale. Piuttosto, possiamo distinguere tre fasi in cui i partiti di estrema destra hanno avuto particolare successo.

La prima è quella dell'estremismo di destra postbellico, che ebbe inizio nel 1945 e gradatamente si estinse negli anni cinquanta. Come si può vedere, i risultati elettorali più notevoli si registrarono nel 1950-51. Nel 1950 la DG ricevette il 14,7\% dei voti e sedici seggi alle elezioni del parlamento del Baden-Württemberg, nonché, in una lista comune con la Unione degli Espulsi e dei Rifugiati (BHE), il 12,3\% e sei seggi alle elezioni del parlamento della Baviera (Stöss 1989, 120)12. Nel 1951 i partiti estremisti di destra (comprese la SRP e la DRP) ottennero il $14,1 \%$ in Bassa Sassonia e il $7,7 \%$ a Brema (Schmollinger 1986d, 2309). Malgrado alcuni notevoli risultati della DRP, il periodo fra il 1952 e il 1958 sembra costituire una fase di declie la DG sei.

12 La lista comune di entrambi i partiti ottenne 26 seggi: la BHE ne ricevette venti 
no. Negli anni del «miracolo economico», l'«opposizione nazionalista» patì insuccessi elettorali, divisioni, rivalità organizzative e personali e isolamento sociale. La sua clientela elettorale si era ridotta a un piccolo nocciolo duro di outsiders antidemocratici (Stöss 1989, 97).

Nei primi anni sessanta si assisté agli sforzi massicci della destra radicale per raccogliere le forze e dar vita a un partito di dimensioni maggiori. L'esito più significativo di tali sforzi fu la formazione della NPD (Schmollinger 1986c, 1923-1926). Il risultato della NPD alle elezioni per il Bundestag del $1965 \mathrm{fu}$ modesto: $2 \%$. Comunque, la prima grande recessione economica dopo il 1949, la disintegrazione della coalizione borghese formatasi nel dopoguerra, la Grande Coalizione di CDU/CSU e SPD a livello federale e di Länder, così come le attività della opposizione extraparlamentare di sinistra, contribuirono all'ascesa della NPD tra il 1966 e il 1968: in questo periodo il partito riuscì a superare la soglia del $5 \%$ nelle elezioni di sette Diete statali ${ }^{13}$. Ma dopo non essere riuscita a ottenere più del 4,3\% dei voti alle elezioni per il Bundestag del 1969, le fortune elettorali della NPD declinarono. Fra il 1972 e il 1986, retrocesse a una quota elettorale vicina allo zero. La CDU/CSU recuperò la maggior parte di quegli elettori che avevano spostato la loro fiducia politica sulla NPD in maniera temporanea fra il 1966 e il 1968. Negli anni settanta la NPD cadde in agonia e $\mathrm{fu}$, talvolta, sorpassata dalla DVU che, almeno in termini di numero di affiliati, nel 1980 divenne la più forte organizzazione di estrema destra nella Repubblica Federale, e dal partito dei $R e$ publikaner (1989).

La terza ondata del voto di estrema destra è stata provocata fondamentalmente dal successo dei Republikaner. Nel 1989, questo partito superò la soglia del $5 \%$ a Berlino e alle elezioni europee (Stöss 1989, 217). Sebbene le elezioni del Parlamento europeo di solito non siano considerate tanto importanti quanto quelle del Bundestag, questa fu la prima volta che una formazione di estrema destra guadagnò più del $5 \%$ dei voti in una elezione su scala nazionale. Nel 1990 l'unificazione tedesca sembrò interrompere l'ascesa dell'estrema destra. Ciononostan-

13 1966: Baviera (7,4\% del voto e 15 seggi parlamentari), Assia (7,9\%, 8 seggi); 1967: Schleswig-Holstein $(5,8 \%, 4$ seggi), Renania-Palatinato $(6,9 \%, 4$ seggi), Bassa Sassonia $(7 \%, 10$ seggi), Brema $(8,8 \%, 8$ seggi); 1968: Baden-Württemberg $(9,8 \%, 12$ seggi). 


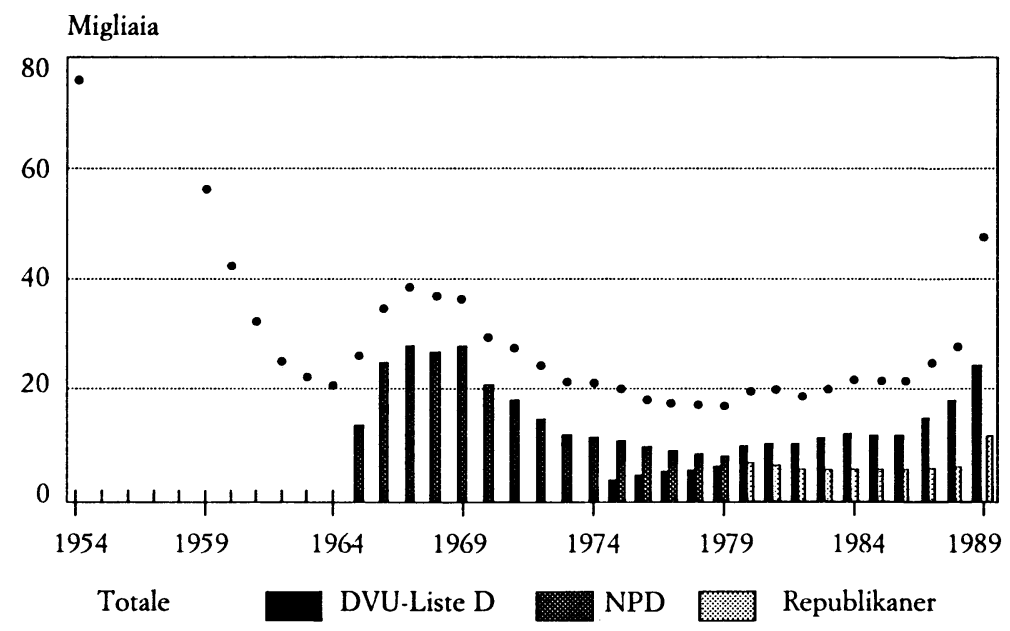

FIG. 2. Iscritti ai partiti di estrema destra in Germania Ovest (1954-1989)

Fonti: 1954-1989: Ministero federale dell'Interno: Verfassungsschutzbericht; per i Republikaner: Stöss $(1990,54)$.

te, le elezioni del 1992 per le Diete statali in Baden-Württemberg, Schleswig-Holstein e a Brema dimostrarono che l'unificazione tedesca non aveva sferrato un colpo fatale ai Republikaner e alla DVU. Il partito dei Republikaner ottenne il 10,9\% dei voti e quindici seggi alla Dieta del Baden-Württemberg. Alle elezioni di Brema (1991) e dello Schleswig-Holstein (1992) la DVU ottenne il 6,2 e il 6,3\% rispettivamente e sei seggi parlamentari nelle Diete di entrambi i Länder (Roberts 1992, 344; May 1992, 123).

Se diamo uno sguardo al numero degli iscritti alle organizzazioni di estrema destra, emerge un modello ciclico simile. La fig. 2 mostra un modello in gran parte analogo a quello osservato nella fig. 1 per quanto riguarda il sostegno elettorale dei partiti di estrema destra. La linea punteggiata segna il totale degli iscritti di tutte le organizzazione di estrema destra dal 1954 al 1989. Le colonne si riferiscono alle organizzazioni più importanti ${ }^{14}$. Il numero complessivo dei membri inquadrati in gruppi

${ }^{14}$ Si considera qui la DW come un partito, benché non sia diventata un partito politico regolare sino al 1987. 
e partiti di estrema destra diminuì rapidamente dal 1954 ai primi anni sessanta. La ripresa fra il 1964 e il 1967 fu dovuta in larga misura alla crescente attrattiva esercitata dalla NPD, mentre la tendenza calante dopo il 1967 è correlata anche con una perdita continua di affiliati della NPD. Fra il 1978 e il 1988 il numero degli estremisti organizzati di destra crebbe di circa il $60 \%$. Gli aumenti più notevoli ebbero luogo nel 1979-80 e fra il 1986 e il 1988. Per lo più, sembrano essere una conseguenza della crescente forza organizzativa della DVU. Nonostante vi sia stato un processo di concentrazione del consenso a favore della DVU, l'estrema destra tedesco-occidentale ha mantenuto nel tempo una delle sue caratteristiche tradizionali: il suo alto grado di frazionalizzazione. Fra il 1970 e il 1989 il numero di organizzazioni di estrema destra registrate dal Ministero Federale degli Interni ha oscillato fra un minimo di 62 (1973) e un massimo di 91 (1975) (Bundesministerium des Innern 1969/701992).

A prescindere dalle organizzazioni tradizionali dominanti, la NPD e la DVU, i piccoli gruppi militanti hanno avuto un tasso di crescita considerevole dagli anni settanta. Dal 1975 al 1989, i loro iscritti sono aumentati cinque volte. In cifre assolute, comunque, sono rimasti una quantità insignificante. Nel 1989 il Ministero Federale degli Interni ha contato 23 organizzazioni con 1500 sostenitori. Nel 1991 il numero delle organizzazioni è aumentato a trenta per un totale di 2100 iscritti. I tassi di incremento più forte si osservarono fra il 1975 e il 1979. Dopo un periodo di stagnazione nei primi anni ottanta, che si dovette probabilmente alla messa al bando nel 1983 di molti gruppi militanti e alla messa in stato d'accusa dei loro dirigenti, $i$ gruppi di destra a carattere paramilitare sono andati incontro a un altro periodo di crescita nel 1987 e, specialmente, dopo l'unificazione. In seguito all'escalation di fenomeni violenti occorsi nel 1991-92, nel dicembre 1992 il Ministero Federale degli Interni ha messo al bando due dei gruppi più famigerati, l'Alternativa Tedesca e il Fronte Nazionalista.

Oltre a questi tre cicli elettorali e organizzativi, si possono individuare una quarta e persino una quinta fase di attività dell'estrema destra. Queste fasi sono caratterizzate dal crescente ricorso a comportamenti illegali e alla violenza. La fase 1977-85 un periodo catastrofico per l'area dell'estrema destra sotto il profilo elettorale - venne caratterizzata dall'aumento dell'aggressività dei gruppi neonazisti. La fig. 3 illustra il numero di 


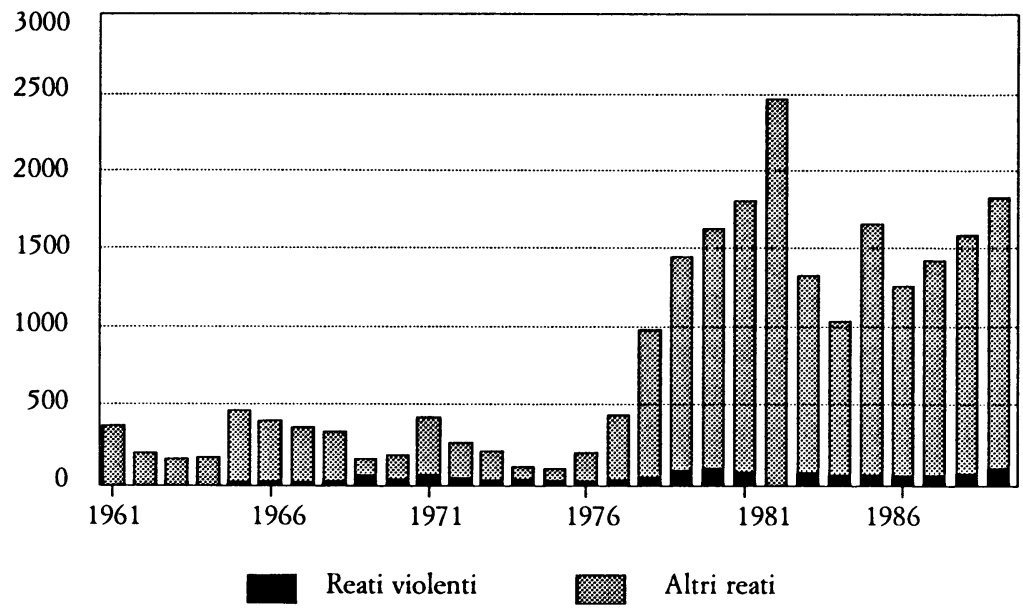

FIG. 3. Reati commessi da estremisti di destra in Germania Ovest (1961-1989)

Fonte: Ministero Federale dell'Interno: Verfassungsschutzberichte.

reati commessi da estremisti di destra secondo quanto registrato dal Ministero Federale degli Interni. Le parti inferiori delle colonne indicano $i$ reati violenti e le parti superiori le minacce di violenza e i reati senza violenza, come gli insulti o le scritte razziste $o$ antisemite e simili (Bundesministerium des Innern 1989, 142-143). La fig. 3 dimostra che il numero complessivo annuale di reati registrati commessi da estremisti di destra è aumentato senza interruzioni fra il 1976 e il 1982. Fra il 1982 e il 1990 questo numero è rimasto invariato, sebbene su un livello considerevolmente più alto che negli anni sessanta e settanta. Fino al 1990 solo una minima proporzione di tutti i reati commessi dagli estremisti di destra ha avuto carattere violento. Nella seconda metà del 1991 e nella prima metà del 1992, tuttavia, si è avuta una tremenda ondata di aggressioni violente, specialmente contro ostelli per profughi in cerca d'asilo. Nella Germania Occidentale, il numero delle aggressioni violente è quasi quadruplicato, passando dai 270 casi del 1990 ai 990 del 1991. Nella Germania Orientale, l'Ufficio Federale per la Protezione della Costituzione ha contato nel 1991493 aggressioni violente (Bundesministerium des Innern 1992, 76; Roberts 1992, 337. 338). 


\section{Le radici dell'elettorato di destra}

I partiti estremisti di destra diventano politicamente rilevanti non appena influenzano l'aritmetica delle maggioranze e delle coalizioni nel sistema decisionale. La caduta della Repubblica di Weimar lo illustra bene. Alcuni sondaggi hanno mostrato ripetutamente che vi è, nell'elettorato tedesco occidentale, un potenziale conservatore-autoritario latente che è assai più alto delle quote di voti ottenute dai partiti di estrema destra nelle diverse tornate elettorali (Fisher 1974, 146; Sinus 1981; Falter e Schumann 1988, 99-100). Le cifre variano a seconda della concettualizzazione degli atteggiamenti di estrema destra usata nei vari sondaggi di opinione. Fisher $(1974,146)$ e Sinus $(1981)$ sostengono che questo potenziale si aggira fra $1{ }^{\prime} 8 \%$ e il $15 \%$. Falter e Schumann $(1988,99-100)$ fanno uso di una definizione più restrittiva, vale a dire la posizione ideologica su un continuum sinistra-destra da 1 a 10 come quello impiegato nei sondaggi dell'Eurobarometro. Questi autori stimano nel 3,7\% la dimensione dell'elettorato di estrema destra (ossia, coloro che occupano la posizione 10 su questo continuum). «Nelle passate elezioni, alcuni di questi uomini e donne si sono astenuti; altri hanno votato per una varietà frammentata di gruppi di destra; altri ancora, per una mancanza di alternative accettabili, hanno sostenuto la destra della CDU o della FDP» (Fisher 1974, 146). A certe condizioni, tuttavia, i partiti di protesta dell'estrema destra sono in grado di mobilitare questo potenziale. Perciò vi è un pericolo di destabilizzazione. La questione cruciale è: a quali condizioni questo potenziale latente può diventare una minaccia manifesta? La discussione che segue costituirà un tentativo di identificare alcune di queste condizioni.

Una delle spiegazioni più influenti dell'estremismo di destra è l'ipotesi del radicalismo della classe media di Seymour Martin Lipset. Secondo Lipset «ogni strato sociale principale ha contemporaneamente espressioni politiche democratiche ed estremiste»; il fascismo e l'estremismo di destra sono, fondamentalmente, movimenti di classe media (Lipset 1960, 131-134). Gli elettori della classe media, specialmente i piccoli imprenditori, gli agricoltori e gli artigiani indipendenti tendono a radicalizzarsi, se si sentono minacciati da processi di concentrazione e di centralizzazione di larga portata - sviluppi caratteristici delle moderne società industriali - e da crisi economiche specifiche (Lipset 1960, 134-152). 
Come spiegazione dell'ascesa del partito nazionalsocialista in Germania, la tesi di Lipset è stata respinta da diversi punti di vista (Winkler 1972; Falter e Zintl 1989, 165). Neppure il radicamento sociale dell'estremismo di destra nella Repubblica Federale Tedesca offre prove evidenti in sostegno della sua ipotesi. Purtroppo non disponiamo di alcun dato di sondaggio sull'elettorato dei partiti di estrema destra dei primi anni cinquanta. Per quel che riguarda questo periodo, i dati aggregati sembrano sostenere l'ipotesi di Lipset, sebbene col pericolo della fallacia ecologica. I partiti di estrema destra degli anni cinquanta ebbero particolare successo nelle circoscrizioni rurali e nelle piccole città ove le vecchie classi medie erano sovrarappresentate (Schmollinger 1986b, 1173-1174; Schmollinger 1986d, 2330; Stöss 1986b, 895-896). Analogamente, la maggior parte delle roccaforti della NPD erano dominate da una popolazione agricola e di classe media (Schmollinger 1986c, 1955-1956). Dati di sondaggio sugli elettori della NPD confermano che la NPD era sovrarappresentata fra i lavoratori indipendenti (coltivatori diretti, piccoli imprenditori e professionisti), ma bisogna anche sottolineare che otteneva un'alta quota di voti da operai non qualificati, in specie di piccole imprese (Liepelt 1967, 241-247). Nonostante sia fin qui compatibile con la tesi di Lipset, il riscontro empirico non riesce invece a rendere conto delle cause del voto al partito dei Republikaner. Nel 1989 si è riscontrato che i membri delle vecchie classi medie erano meno inclini a sostenere partiti di estrema destra, come i Republikaner, di quelli della classe operaia. Fra i Republikaner, le classi sociali sovrarappresentate in misura più impressionante risultarono essere quelle degli operai semplici e specializzati (Niedermayer 1990, 572-573; Infratest 1989, 26; Roth 1990a, 35) ${ }^{15}$.

Benché la tesi specifica di Lipset abbia un valore limitato per spiegare la vasta attrattiva elettorale esercitata dai Republikaner nel 1989, l'assunto generale che sottende, e cioè che $\mathrm{i}$ processi di modernizzazione economica e sociale causano la deprivazione economica di certi individui o gruppi tale da renderli, a loro volta, più attratti dai partiti di estrema destra, potreb-

15 Questo risultato ha come riferimento quei rispondenti che dichiararono di essere intenzionati a votare per il partito dei Republikaner. Comunque, anche Bauer e Niedermayer $(1990,25)$ hanno rilevato che, fra coloro che si considerano di estrema destra in termini di ideologia e opinioni, le vecchie classi medie sono abbondantemente sovrarappresentate. Ciò confermerebbe la tesi di Lipset. 
be pur sempre essere considerata come una spiegazione plausibile. Chi propone questa ipotesi sostiene che la maggioranza degli elettori di estrema destra sia costituita dai «perdenti» del processo di modernizzazione sociale (cfr. per esempio Stöss 1989, 235-236). In questo contesto si può far corrispondere il concetto di «deprivazione», o frustrazione delle aspettative, a un processo oggettivo di marginalizzazione o a sentimenti soggettivi di insicurezza, o alla paura di un'attesa perdita di status sociale (Niedermayer 1990, 573). Inoltre, pare logico che fenomeni di deprivazione relativa diventino più virulenti in tempi di crisi economica. Sulla base dei dati aggregati disponibili per gli anni cinquanta, sembra plausibile che la deprivazione relativa possa costituire una variabile esplicativa importante per gli anni dell'immediato dopoguerra. La DReP's, la DRP, la SRP e la DG mobilitarono la protesta dei disoccupati, degli espulsi e degli ex nazionalsocialisti politicamente sradicati. Kaltefleiter ha stimato, sulla base di dati aggregati, che nel 1949 la metà degli elettori del Partito di Destra Tedesco (DReP) in Bassa Sassonia era formata da rifugiati ed espulsi. Inoltre, la DReP ebbe anche successo fra le popolazioni native, laddove la disoccupazione era relativamente alta. La percezione pessimistica della situazione economica personale e generale sembra aver contribuito ai successi della DRP nelle elezioni del 1959 della Renania-Palatinato (Kaltefleiter 1968, 134-136). Anche se non abbiamo nessun dato preciso sugli elettori della DG nella Germania meridionale, pare che questo partito abbia rivolto la propria propaganda agli stessi gruppi sociali a cui facevano appello DReP e SRP (Fisher 1974, 136, vedi sopra) ${ }^{17}$. Si può spiegare il declino dei voti per i partiti di estrema destra nella seconda metà degli anni cinquanta a partire da queste premesse, dopo il 1951 le migliori prospettive economiche cominciarono a ridurre la protesta dell'estrema destra contro la deprivazione economica. Di ciò, il principale beneficiario fu la CDU/CSU (Fisher 1974, 135).

16 Il Partito Conservatore Tedesco - Partito Tedesco di Destra (DReP, Deutsche Konservative Partei-Deutsche Rechtspartei) era un partito nazionalista conservatore, nel solco della tradizione del Partito Nazional-Popolare Tedesco di Weimar (DNVP, Deutschnationale Volkspartei), che durò dal 1946 al 1950 (Schmollinger 1986a).

17 È chiaro che la propaganda di un partito non dovrebbe essere messa alla stregua delle motivazioni di voto dei suoi elettori. Tuttavia, in assenza di dati sull'elettorato della DG, abbiamo preso questi elementi informativi come surrogati delle motivazioni, tenendo a mente i rischi di errore. 
Per la seconda metà degli anni sessanta, la situazione economica, e i fenomeni di deprivazione relativa che da questa situazione erano scaturiti, possono essere interpretati come una condizione sufficiente, ma non necessaria, della crescita elettorale della NPD in quel momento (Liepelt 1967, 265). La simpatia per la NPD non rimase confinata a coloro i quali erano stati più colpiti dalla recessione del 1966-67. Al contrario, gli elettori con redditi più alti furono più inclini a simpatizzare con la NPD di chi aveva introiti minori. La variabile cruciale non era una situazione economica oggettiva, ma le aspettative economiche per il futuro. La NPD riscosse particolare successo fra le persone le cui aspettative sui futuri sviluppi dell'economia erano pessimistiche (Liepelt 1967, 255-256). Si potrebbe quindi spiegare il declino della NPD dopo il 1969 in ragione della ripresa economica cominciata nel 1968. Tuttavia, i disastrosi risultati elettorali della NPD durante le due più grandi recessioni degli anni settanta dimostrano che lo stato di forma dell'economia e i sintomi di deprivazione individuale che ne risultano rappresentano solo una condizione sufficiente, ma non necessaria, dell'incremento elettorale dei partiti di estrema destra.

Neppure l'ondata ascendente dei voti ai Republikaner nel 1989 può essere spiegata agevolmente in base alla deprivazione relativa. Benché negli anni ottanta si sia assistito a uno sviluppo economico bifronte, ossia a una crescita economica in parallelo con una disoccupazione di lungo termine, che ha allargato il divario fra la maggioranza di chi sta economicamente bene e una minoranza formata da persone afflitte da disagi economici, i sondaggi non sono in grado di individuare una relazione statistica chiara fra reddito e voto per il partito dei Republikaner. Per quanto riguarda il reddito familiare e la proprietà della casa, i sostenitori dei Republikaner nel 1989-90 risultano ampiamente rappresentativi della popolazione nel suo complesso (Infratest 1989, 27; Niedermayer 1990, 574). Cionondimeno, il voto per i Republikaner fu parzialmente motivato dalla percezione di una minaccia dello status economico e sociale degli individui, dal sentirsi menomati politicamente ed economicamente (Roth 1990b, 7-8). Sembra esserci una correlazione negativa tra la soddisfazione per la propria situazione economica individuale e le aspettative future da una parte e il voto per i partiti di estrema destra dall'altra. I sentimenti di insoddisfazione e le paure per i futuri sviluppi sono molto diffusi nell'elettorato dei Republikaner (Niedermayer 1990, 572-574). Dunque, almeno a 
livello soggettivo, vi sono alcuni riscontri empirici a favore della tesi della deprivazione relativa.

Nella loro analisi del successo della NPD negli anni sessanta, Scheuch e Klingemann hanno cercato di spiegare l'esistenza di un potenziale di estremismo di destra come fenomeno che è presente in tutte le società industriali; questi autori considerano l'estremismo di destra «una condizione patologica "normale"» (Scheuch e Klingemann 1967, 29).

In ogni società industriale interessata da un rapido cambiamento, le tensioni sono prodotte in vari settori e a vari livelli del sistema sociale. Queste tensioni assumono spesso la forma di un conflitto tra valori orientati al gruppo primario e $\mathrm{i}$ requisiti funzionali delle istituzioni secondarie. L'individuo viene a contatto di questo conflitto nelle richieste contraddittorie delle situazioni della vita quotidiana. [...] Una forma di adattamento a queste condizioni ambientali è la rigidità o una mentalità chiusa. Perciò, se la struttura affettiva e cognitiva dell'individuo è influenzata dalla struttura sociale in questa maniera, ogni società industriale deve avere un numero considerevole di persone con sistemi di credenze di questo tipo (Scheuch e Klingemann 1967, 29).

Questo argomento è stato elaborato da Falter e Schumann. Essi affermano che specialmente «molti elettori più anziani e una proporzione considerevole dei più giovani non posseggono gli strumenti analitici o cognitivi necessari per affrontare le mutevoli richieste della società moderna: i gruppi più vecchi perché possono aver acquisito certi abiti mentali e una certa disposizione interpretativa di stampo autoritario negli anni della loro prima formazione intellettuale e sociale, e anche a causa della tendenza naturale a divenire meno flessibili e tolleranti con l'età; i più giovani, perché possono appartenere a una "generazione senza futuro" con alti livelli di disoccupazione e una generale incertezza circa le prospettive per il futuro». Entrambi i gruppi «mostrano spesso una tendenza a sviluppare interpretazioni semplicistiche di un mondo complesso e sconcertante. Per affrontare le richieste della società moderna, essi tendono - inconsciamente, è chiaro - a sviluppare certi meccanismi psicologici di difesa come una rigidità cognitiva, una propensione per la stabilità e interpretazioni nette ma inadeguate del mondo. Questi meccanismi di difesa tendono, a loro volta, in certe circostanze, a favorire l'estremismo di destra» (Falter e Schumann 1988, 107-108).

In modo simile, Klingemann e Pappi $(1972,51)$ sottolineano l'importanza dell'istruzione, oltre che dell'età, quale variabile esplicativa: a un più alto livello di istruzione corrisponde una 
trasmissione più esplicita e intensa di quei valori che sono caratteristici di un certo sistema politico. Con questa ipotesi di socializzazione legata alla specificità del sistema, Klingemann e Pappi hanno cercato di spiegare perché il sostegno alla NPD fosse relativamente più forte fra le persone più anziane con uno standard educativo relativamente più alto, che furono scolarizzate per la maggior parte nel periodo della dittatura nazionalsocialista, mentre nel sistema democratico formatosi dopo il 1945 un livello di scolarità più alto ha ovviamente favorito la trasmissione dei valori democratici e ha quindi ridotto la probabilità che gli individui votino per partiti di estrema destra. La composizione sociale dell'elettorato della NPD sembra suffragare l'assunto teorico sopraccitato. Dati provenienti da sondaggi rappresentativi del 1970-71 mostrano che la ricettività generale dell'estremismo di destra diminuisce al crescere del livello di scolarizzazione. Tuttavia, fra i soggetti intervistati con un livello di istruzione più alto, le coorti di età con più di 46 anni rivelavano di avere un'inclinazione al voto per la NPD molto maggiore di coloro che avevano un'età inferiore ai 46 anni (Klingemann e Pappi 1972, 50-51).

La composizione dell'elettorato potenziale dei Republikaner per età e livello di istruzione sembra contraddire la tesi Klingemann-Pappi solo a prima vista. Nel 1989, il 51\% dei sostenitori dei Republikaner disponeva di un titolo di studio di livello elementare e di esperienze di apprendistato. La quota rispettiva, nel totale della popolazione, era del 38\%. Solo l' $8 \%$ dell'elettorato di questo partito si collocava nel gruppo con il livello di istruzione più alto, con un titolo di Abitur o un diploma universitario. Questi risultati variano se si controlla la variabile «età». Gli elettori dei Republikaner con meno di cinquant'anni appartengono tendenzialmente a strati sociali caratterizzati da livelli di istruzione più bassi. Fra gli elettori dei Republikaner con oltre sessant'anni, le differenze di istruzione in buona misura scompaiono (Roth 1989, 12-13). Ciò è in sintonia sia con i risultati di Klingemann e Pappi che con quelli di Falter e Schumann. Per avere una spiegazione strutturale alternativa si potrebbe ricorrere alla teoria della società di massa di Kornhauser. Secondo Kornhauser le differenze nella ricettività dei simboli e dei leader di massa «sono dovute principalmente alla forza dei legami sociali, e non all'influenza della posizione di classe o a qualunque altro status sociale in sé» $(1960,237)$. Le società industriali moderne hanno contribuito all'«alienazione so- 
ciale» e hanno atomizzato i gruppi sociali tradizionali. In un sistema pluralista democratico, i gruppi sociali mediano fra élites e masse e, così facendo, riducono l'alienazione sociale potenziale. Nella misura in cui questi gruppi hanno perso la loro capacità di soddisfare le loro funzioni di mediazione, i loro membri sono diventati socialmente isolati; di conseguenza, si sviluppa una società di massa in cui è più alta la ricettività dei leader totalitari.

Si è sostenuto, nella teoria della società di massa, che degli stretti legami con ambienti sociali come quelli offerti dalla Chiesa cattolica o dai movimenti sindacali fungono tendenzialmente da barriere contro la propaganda dei movimenti di estrema destra. Per ciò che riguarda la Chiesa cattolica, la tesi è quantomeno plausibile per la NSDAP dei primi anni trenta; $i$ legami con il movimento sindacale, d'altra parte, non sembrano costituire necessariamente una barriera effettiva (Falter 1982, 457-458). Per gli elettori dei partiti di estrema destra dei primi anni cinquanta, si potrebbe dimostrare che la DReP, la DRP e la SRP riscuotevano i maggiori successi nelle regioni protestanti con una struttura agricolo-rurale e nelle piccole città protestanti, ossia nelle aree dove né la Chiesa cattolica né i sindacati erano in grado di impiantare un ambiente dominante (Schmollinger 1986b, 1173-1174; Schmollinger 1986d, 2330; Kaltefleiter $1968,136)$. Anche la NPD riceveva più consensi nelle regioni con una percentuale maggiore di protestanti (Schmollinger 1986c, 1955-1956). Più erano stretti i legami con la Chiesa Cattolica, più era bassa la probabilità che un elettore sostenesse la NPD. Si è accertato che l'affiliazione ai sindacati costituisce una barriera analoga contro il voto di estrema destra. Ciò non significa che stretti legami con una di queste organizzazioni intermedie abbiano offerto una protezione assoluta contro il voto alla NPD, ma è certo che contribuirono a stabilizzare le clientele elettorali della SPD e della CDU/CSU nei momenti di tensione economica e politica (Liepelt 1967, 245). Fra gli elettori dei Republikaner, non vi è quella sovrarappresentazione di protestanti riscontrata tradizionalmente nell'elettorato dei partiti di estrema destra. Ciò si spiega facilmente col fatto che il partito dei Republikaner è essenzialmente un fenomeno bavarese e meridionale, di quelle regioni cioè in cui la percentuale di elettori cattolici è sproporzionatamente più alta. Se però si considera il grado di identificazione con la Chiesa cattolica, il modello di comportamento elettorale atteso riemerge; ossia, quanto più 
sono stretti i legami di una persona con la Chiesa cattolica, tanto minori sono le probabilità che voti per i Republikaner. Di contro, l'iscrizione ai sindacati non costituisce più una barriera contro i partiti di estrema destra. Anzi, la probabilità che i membri dei sindacati votino per i Republikaner è persino più alta di quanto non succeda nella popolazione non sindacalizzata (Roth 1990b, 5; Roth 1989, 14; Niedermayer 1990, 579).

Le teorie di cui si è fatta menzione non sono per lo più in grado di spiegare e neppure di rendere conto delle due caratteristiche più notevoli degli elettorati dei partiti di estrema destra alla fine degli anni sessanta e degli anni ottanta: le differenze di genere e regionali. Quasi due terzi degli elettori potenziali della NPD nel 1965-66 e degli elettori potenziali dei Republikaner del 1989 erano maschi. Ed entrambi i partiti, tradizionalmente, traggono la maggior parte del loro sostegno dalla Germania meridionale, vale a dire dalla Baviera e dal Baden-Württemberg (Liepelt 1967, 241; Roth 1990b, 3-6). L'assunto secondo cui gli uomini sarebbero più interessati delle donne alle vicende politiche correnti e che le donne generalmente tenderebbero a reagire più cautamente ai nuovi sviluppi politici (Roth 1989 11-12) non regge alle verifiche cross-national (Niedermayer 1990, 577578). La forza relativa della NPD e dei Republikaner nella Germania del Sud può essere spiegata con qualche plausibilità sulla base del livello relativamente alto di organizzazione raggiunto da questi due partiti in Baviera e Baden-Württemberg. Inoltre, il leader carismatico dei Republikaner, Schönhuber, è bavarese, e il partito sta utilizzando molto, nelle sue campagne e nelle sue adunate, simboli specificamente bavaresi (Roth 1990b, 5-6).

Tutte le teorie abbozzate fin qui spiegano, in misura differente, l'esistenza di un potenziale radicale di destra nel popolo tedesco. Queste teorie hanno contribuito in misura notevole alla comprensione delle radici sociali dell'estremismo di destra. Purtuttavia, il loro potere esplicativo è ridotto se si vuole sapere in quali circostanze il potenziale estremistico di destra rimanga latente e a quali condizioni diventi invece manifesto. Alcuni studi sulle cause del sostegno alla NPD e ai Republikaner hanno dimostrato che, se si vuole comprendere l'ondata ascendente del voto per i partiti di estrema destra nella seconda metà degli anni sessanta e nel 1989, devono essere presi in considerazione i fattori politici, in aggiunta a quei fattori elaborati nelle analisi socio-strutturali del voto. Rispetto al totale dell'elettorato, una quota sproporzionatamente alta di elettori della NPD e dei $R e$ - 
publikaner ha espresso una fortissima insoddisfazione per il funzionamento del sistema politico, la sua capacità di affrontare le sfide economiche e i partiti politici più solidi (Liepelt 1967, 262-263; Niedermayer 1990, 580-581). Questo punto induce a spostare la nostra attenzione sulle variabili politiche dal «lato dell'offerta» del processo politico, come illustrato nella fig. 4 . Data una «domanda» più o meno costante di schemi interpretativi di estrema destra (8-15\% dell'elettorato tedesco-occidentale, cfr. Fisher 1974, 146; Sinus 1981), i fattori politici dovrebbero aiutare a spiegare le variazioni di manifestazione dell'estremismo di destra nel comportamento politico.

Questa prospettiva supply-side è rafforzata dall'esperienza post-unificazione. Il governo Kohl ha esibito un alto grado di competenza nel condurre i negoziati che hanno portato ai trattati di unificazione. Esso è riuscito a dominare la questione politica nazionale lasciando poco spazio ai partiti estremisti di destra. Però, non appena è svanita l'euforia per l'unificazione tedesca ed è emersa la dimensione dei problemi economici, fiscali e sociali, i governi di Bonn e dei Länder sono stati incapaci di proporre risposte credibili - su come ristrutturare l'economia improduttiva della Germania Est, evitare la disoccupazione di massa all'Est, finanziare le rimesse di denaro da Ovest ad Est e affrontare le ondate di immigrazione dall'Europa orientale e dai paesi del Terzo Mondo. Su questo sfondo, i partiti di estrema destra sono stati in grado di riguadagnare terreno, beneficiando dell'insoddisfazione generale e reimpossessandosi della tematica nazionalista - per lo più con le loro vigorose campagne antiimmigrazione e antiprofughi.

\section{Un modello multivariato}

In una sinossi analitica abbiamo elencato sei variabili («variazioni nello spazio del sistema dei partiti», ossia composizione della coalizione di governo, «crisi economica», «spazio tematico», «repressione governativa», «fazionalismo dell'estrema destra» e «leadership carismatica») e classificato il materiale descrittivo. Allo stesso tempo abbiamo sviluppato il seguente modello causale, rudimentale ma parsimonioso (fig. 4).

$\mathrm{La}$ «presenza dei conservatori nelle coalizioni di governo» è ritenuta la variabile causale necessaria più importante per determinare il successo elettorale di un partito di estrema destra. Per 


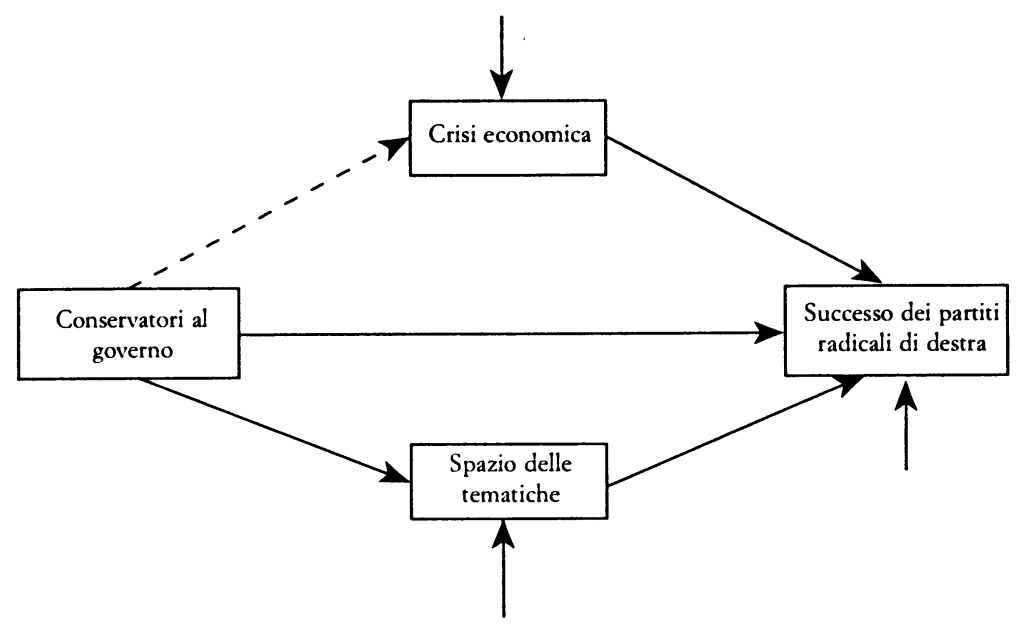

FIG. 4. Le tre variabili chiave per la spiegazione della forza dei partiti di estrema destra

contro, la «crisi economica» vale solo come variabile sufficiente. Lo «spazio tematico», in particolare il monopolio di una tematica nel lungo periodo, tuttavia, è vicino ad avere valore di condizione necessaria. Questo modello è stato altrove discusso in maniera teoricamente ed empiricamente più dettagliata (Zimmermann e Saalfeld 1994). Una verifica empirica preliminare e indiretta condotta su tutte le elezioni a livello di Länder nella Germania occidentale porta a risultati incoraggianti.

Nel caso della prima ondata dell'estremismo di destra tedesco-occidentale, ci fu un'apertura nello spazio del sistema dei partiti, con i conservatori (la CDU/CSU e altri partiti minori come il Partito Tedesco) nella coalizione di governo, accompagnata da problemi economici quali la ricostruzione postbellica e l'integrazione dei rifugiati tedeschi in alcune zone della Bassa Sassonia, dell'Alta Franconia, dell'Assia e del Palatinato Occidentale. Tuttavia, sussistevano quattro fattori che deprimevano la forza di qualunque partito di estrema destra: 1) la destra radicale era frazionata fin dall'inizio (DReP, DRP, SRP, DG, e molti partiti dissidenti; cfr. Stöss 1986b; 1989); 2) dal 1950 in poi, la BHE, che optava per politiche degli interessi di carattere pragmatico, si rivelò essere una forte concorrente per quella destra radicale; 3) nel 1952, la repressione del governo colpì la $\mathrm{SRP}$, e lo stile di leadership, carismatico e semiautoritario, di 
Adenauer tenne sotto controllo alcuni fra gli elettori di destra più radicali; 4) il boom economico si rivelò così forte che, fino alla fine degli anni cinquanta, la destra radicale scomparve, quando ancora era al governo un partito conservatore.

Nella seconda ondata di sfide della destra estremista, la NPD emerse come il partito più forte. Il suo sostegno era diffuso più uniformemente in tutte le aree dell'intero elettorato. Gli elettori radicali di destra non provenivano più da zone geografiche particolari, per quanto certe influenze ambientali fossero ancora notevoli in regioni come la Bassa Sassonia e la Media Franconia. La grande coalizione del 1966 e la prima crisi economica rilevante nella storia della Repubblica Federale aprirono a destra lo spazio del sistema dei partiti e dettero alla NPD una spinta in più in virtù del voto di protesta contro la situazione economica. Il partito si autodefinì «diverso» dai «partiti autorizzati di Bonn». Oltre alla questione nazionale sempre serpeggiante e alla crisi economica, tuttavia, la NPD non cavalcò nessun tema specifico. Lo sbarramento elettorale del $5 \%$ nelle elezioni federali del 1969 fece il resto per la NPD. In quel momento, mancava una leadership politica carismatica tanto ai conservatori (CDU/CSU) quanto agli estremisti di destra. Perciò, malgrado un livello relativamente basso di frazionalizzazione della destra radicale, in un momento di crisi economica su vasta scala, e in presenza di una grande coalizione a guida conservatrice, la NPD non ebbe successo, per cause in primo luogo politiche (la nuova vigorosa opposizione formata dalla CDU/ CSU nel 1969) e in secondo luogo economiche, con il superamento del momento più cupo della recessione nel 1968 (Liepelt 1967; Roth 1989, 16). Ancora una volta sembra che il fattore dominante fosse di natura politica, ossia quale partito o coalizione di partiti era al governo. Nonostante in questa seconda fase la frazionalizzazione della destra radicale fosse più bassa, alla fine il suo destino fu simile.

Così come la seconda ondata di estremismo di destra fu diversa dalla prima (minor frazionalizzazione, maggior spazio di manovra politica per l'esistenza di una grande coalizione al governo), la terza ondata è diversa dalla seconda. La nostra variabile principale, tuttavia, fa sentire di nuovo i suoi effetti, con la sola differenza che la coalizione di governo non è composta dai due partiti maggiori, CDU/CSU e SPD. Tuttavia, come dimostra l'alto numero di ex elettori della SPD che hanno votato o espresso simpatia per i Republikaner, l'opposizione esercitata 
dalla SPD non è percepita come un'alternativa credibile ai partiti conservatori. Gli elettori della CDU/CSU che hanno perso il loro attaccamento per il loro vecchio partito devono guardare altrove, se vogliono esprimere la loro insoddisfazione. La SPD non si occupa della questione dell'immigrazione, della legge e dell'ordine, e del nazionalismo nel modo desiderato dagli elettori di estrema destra. Ciò è in netto contrasto con quanto succedeva negli anni cinquanta, quando la SPD e la FDP facevano consapevolmente appello all'area nazionalista che si sentiva negletta dalla politica di integrazione occidentale promossa da Adenauer in luogo della immediata riunificazione tedesca. Rispetto al periodo $1966-68$, lo scenario economico generale fu positivo fino al 1990, a dispetto di uno sviluppo economico a due velocità (per esempio, agricoltura contro industrie ad alta tecnologia). Almeno per il periodo fino a metà del 1991, non si può considerare la situazione economica come una condizione sufficiente per l'ascesa del partito dei Republikaner e il sostegno elettorale ottenuto dalla DVU. C'è e probabilmente resterà, tuttavia, una certa disoccupazione strutturale che colpisce nella Germania occidentale le persone meno istruite, meno qualificate professionalmente e più anziane. Ovviamente, questi problemi strutturali tendono ad essere assai più seri nella Germania dell'Est. Quindi, non sono necessariamente le circostanze economiche, che, fino al 1991, erano buone per una vasta maggioranza della popolazione tedesco-occidentale, ma le paure soggettive e le aspettative negative a contribuire a creare simpatie per l'estrema destra. Ciò rende comprensibile perché possano porsi al centro dell'attenzione le questioni del diritto d'asilo, della presenza di stranieri provenienti dall'Europa dell'Est e dal Mediterraneo, e la competizione economica per l'alloggio e per il lavoro. L'attenzione rivolta a questi temi funge da capro espiatorio dei problemi immaginati e attesi, e solo su di essi gli elettori e i simpatizzanti dei Republikaner sono d'accordo e danno del loro partito un giudizio più positivo in termini di competenza di quanto non sia riguardo alle capacità di guidare l'economia o di garantire la sicurezza sociale (Infratest 1989).

La maggiore differenza rispetto alle precedenti ondate di estremismo di destra sembra essere l'accento messo sulla presenza degli stranieri nel paese (è vero però che i sentimenti antiamericani furono rilevanti nella propaganda di estrema destra della fine degli anni quaranta e dei primi anni cinquanta). Tuttavia, alla collocazione permanente dei Republikaner o della 
DVU sulla scena parlamentare si frappongono ostacoli severi, come la frazionalizzazione della destra radicale, la scomparsa della «visione» della unificazione tedesca, la mancanza di un numero sufficiente di leader competenti e politicamente uniti e forse anche la soglia del 5\% della legge elettorale tedesca. Nonostante questi partiti abbiano tratto beneficio dalle paure e dalle tensioni create dal massiccio afflusso di profughi fino al 1990 , il loro sostegno è diffuso ed essi non catturano ambienti sociali definiti come sarebbe necessario per tenere alte le sorti di un nuovo partito di protesta (Roth 1989, 19).

Quali sono, dunque, le prospettive dell'estremismo di destra in Germania? È la persistenza di milieu favorevoli in Baviera, Alta e Media Franconia, Bassa Sassonia e altrove a richiedere un'attenzione maggiore, benché l'importanza del fattore «forza in un milieu» stia forse diminuendo in paragone alla continuità di risultati dei primi anni cinquanta e financo degli anni sessanta (Liepelt 1967, 268-269). Bertolt Brecht usava la metafora dell'utero che è ancora fecondo: il 13\% (più o meno) della popolazione che condivide i sentimenti e l'ideologia dell'estrema destra (Sinus 1981; Der Spiegel, 27 Febbraio 1989, p. 49; Heitmeyer 1988) costituisce ciò di cui preoccuparsi nel lungo periodo. Costoro formano realmente, per certi versi, un potenziale patologico la cui evocazione è nelle mani di quei conservatori che sono più inclini a sostenere politiche autoritarie di destra. Forse il gruppo più pericoloso in assoluto è quello formato da vecchi conservatori che vanno alla deriva verso l'estremismo di destra, specie se ciò implica cambiamenti istituzionali quali la rottura di intere ali di partito. Non si può escludere del tutto questa possibilità, soprattutto nei nuovi Länder. Il ruolo cruciale giocato dai conservatori è corroborato dai risultati della ricerca politica comparata, ovverosia dal fatto che, in termini di collocazione sulla scala sinistra-destra, «a parte l'Italia, in tutti i paesi, compresi quelli che hanno partiti di destra, i partiti conservatori o democratico-cristiani catturano la maggioranza relativa del potenziale elettorale di estrema destra» (Bauer e Niedermayer 1990,21$)$. Se i partiti conservatori o alcune ali di partito si spostano su posizioni di estrema destra, fanno capolino alcune reminiscenze della fase finale di Weimar. Le probabilità di questo pericolo dipenderanno principalmente, se non esclusivamente, dalla capacità della CDU/CSU di riacquistare credibilità e - come negli anni cinquanta - di recuperare gli elettori di estrema destra. 
$\mathrm{Ci}$ sono, comunque, differenze significative: a differenza di Weimar, i più importanti partiti pro-sistema finora hanno cooperato nel dare risposta alle sfide di destra - malgrado le controversie sui dettagli delle politiche dell'immigrazione e del diritto d'asilo. Due esempi possono bastare in questo contesto. Il primo: il governo federale e i governi dei Länder di entrambi i partiti si sono trovati d'accordo nel porre i Republikaner, in tutto il paese, sotto la sorveglianza dell'Ufficio Federale per la Protezione della Costituzione. Il secondo: alle vaste dimostrazioni di massa contro la xenofobia a Berlino, Monaco, Amburgo, Norimberga e nelle altre principali città della Germania all'inizio del 1993 hanno preso parte, ostentatamente, i leader, i membri e i sostenitori di tutti i più importanti partiti pro-sistema, eccezion fatta per la dirigenza della CSU. Sebbene non si debbano sottostimare o trascurare la violenza di destra, il sostegno elettorale dato alle formazioni di estrema destra e la considerevole disaffezione per i partiti tradizionali, il consenso di base fra tutti i principali partiti pro-sistema costituisce una differenza decisiva tra la prima e la seconda democrazia tedesca.

Sembra che i pericoli potenziali risiedano altrove. Data la posizione marginale dell'estremismo di destra, ci si deve chiedere se la situazione di ghettizzazione di quel segmento sociale del $13 \%$ e l'occultamento forzato dei loro atteggiamenti e sentimenti politici sotto una «spirale di silenzio» prodotta dal sistema democratico (Noelle-Neumann 1986) ${ }^{18}$, potrà aiutare la democrazia sotto il profilo psicologico. Se gli atteggiamenti (e i pregiudizi) politici dell'estrema destra non sono presi in considerazione, dibattuti e, in ultimo, respinti in un contesto locale, è possibile che questi soggetti semplicemente aspettino il momento e il leader giusto per dischiudere i loro sentimenti «repressi». A sostegno di questa conclusione si potrebbero portare gli scoppi di xenofobia e l'incremento d'attività dei partiti di estrema destra nel territorio che costituiva la Germania orientale, dove l'azione dell'estremismo di destra era stata controllata più vigorosamente che ad Ovest. Le risposte meramente giudiziarie e l'elevare a pubblici tabù i sentimenti di destra, senza che queste idee siano messe in discussione in un pubblico di-

${ }^{18}$ Nelle intenzioni di Noelle-Neumann, questo concetto serviva per spiegare la mancanza di espressioni di simpatia per i partiti conservatori nell'opinione pubblica degli anni Settanta. A suo dire, per l'influenza dei mass media in molti casi il clima di opinione era ostile ad un sostegno apertis verbis per i partiti conservatori. 
battito, spingono quegli elementi radicali a coltivare le loro idee in segreto e a far persistere un potenziale pericoloso.

\section{Conclusioni}

Dopo l'unificazione tedesca, la questione del nazionalismo risulta persino più «morta» di quanto non lo fosse già per la grande maggioranza dei tedeschi occidentali (Scheuch 1990, 739). Certo, ci saranno sempre i fanatici di destra che chiedono la restaurazione della Germania entro i suoi confini del 1937, ma costoro formano solo una piccola minoranza. L'estrema destra, in Germania, ha perso il suo tema centrale. Le issues dell'immigrazione e del diritto di asilo, tuttavia, dureranno probabilmente ancora a lungo e potranno costituire un altro fuoco di cristallizzazione dell'estremismo di destra tedesco. Non si può escludere che possano aver luogo ulteriori sommovimenti sociali causati dai processi di unificazione e risanamento economico nella Germania dell'Est. C'è anche la possibilità di un enorme afflusso di rifugiati dalla Russia e dall'Europa Orientale, se il processo di riforma dovesse fallire. Eppure, dati il caos organizzativo della destra politica, l'importantissima questione di un sollecito aggiustamento economico e le previsioni economiche favorevoli a lungo termine, si può dubitare che i partiti di estrema destra siano in grado di ottenere un'affermazione durevole sulla scena politica.

Le issues sono importanti, ma più importante di tutte le altre variabili del modello causale è la posizione dei principali partner coalizionali conservatori nello spazio del sistema dei partiti. Se le formazioni conservatrici sono all'opposizione, le potenzialità dell'estremismo di destra sono virtualmente nulle. $\mathrm{Ma}$ anche se sono al governo, il complesso insieme di fattori che abbiamo illustrato e le specifiche condizioni attuali di queste variabili rendono improbabile che si avveri la profezia di Neubauer riportata nella citazione iniziale. Fino a questo momento si è dimostrata calzante la previsione di Roth $(1989,19)$, secondo la quale i Republikaner non offrivano alcuna risposta adeguata alle questioni economiche e politiche che sollevavano, si affidavano per lo più a voti di protesta, non possedevano alcuna radice solida nella struttura sociale, e i loro voti esibivano un basso grado di fedeltà al partito ed erano cresciuti troppo in fretta per sostenere le proprie organizzazioni. 
Riferimenti bibliografici

Backes, U. (1990), Extremismus und Populismus von rechts: Ein Vergleich auf europäischer Ebene, in «Politik und Zeitgeschichte», n. 46-47, pp. 3-14.

Backes, U. e E. Jesse (1989), Politischer Extremismus in der Bundesrepublik, Bonn, Bundeszentrale für politische Bildung.

Bauer, P. e O. Niedermayer (1990), Extrem rechtes Potential in den Ländern der Europäischen Gemeinschaft, in «Politik und Zeitgeschichte», n. 46-47, pp. 15-26.

Bickerich, W. (1993), Obne Kurs und Kapitän, in «Der Spiegel», n. 19, 19 maggio, pp. 29-35.

Bundesministerium des Innern (varie annate, dal 1969-70), Verfassungsschutzbericht, Bonn, Bundesministerium des Innern.

Deutscher Bundestag (1990), Woche im Bundestag, XX, n. 20, Bonn, Deutscher Bundestag, pp. 5-11.

Dudek, P. e H.G. Jaschke (1984), Entstehung und Entwicklung des Rechtsextremismus in der Bundesrepublik Deutschland: Zur Tradition einer besonderen politischen Kultur, 2 volumi, Opladen, Westdeutscher Verlag.

Falter, J.W. (1982), Radkalisierung des Mittelstandes oder Mobilisierung der Unpolitischen? Die Theorien von Seymour Martin Lipset und Reinhard Bendix über die Wäblerschaft der NSDAP im Lichte neuerer Forschungesergebnisse, in P. Steinbach (a cura di), Probleme politischer Partizipation im Modernisierunqsprozeß, Stuttgart, Klett Cotta, pp. 438-469.

Falter, J.W. e S. Schumann (1988), Affinity Towards Right-Wing Extremism in Western Europe, in «West European Politics», XI, n. 1, pp. 96-110.

Falter, J.W. e R. Zintl (1989), Weltwirtschaftskrise und NSDAP Wablerfolge: Ein Erklärungsversuch mit Hilfe eines «rationalistichen» Ansatzes und ökologischer Regressionsanalysen, in J.W. Falter e K.G. Troitzsch (a cura di), Wablen und politische Einstellungen in der Bundesrepublik Deutschland, Frankfurt am Main, Lang, pp. $122-174$.

Feit, M. (1987), Die «Neue Rechte» in der Bundesrepublik, Frankfurt e New York, Campus.

Fisher, S.L. (1974), The Minor Parties of the Federal Republic of Germany: Toward a Comparative Theory of Minor Parties, The Hague, Martinus Nijhoff.

Frankfurter Allgemeine Zeitung (1991), Ausländerbaß in den neuen Ländern, n. 45, 22 febbraio, p. 8.

Heitmeyer, W. (1988), Rechtsextremistische Orientierungen bei Jugendlichen: Empirische Ergebnisse und Erklärungsmuster einer untersuchung zur politischen Sozialisation, Weinheim e München, Juventa. 
Herz, A. (1975), Soziale Bedingungen für Rechtsextremismus in der Bundesrepublik Deutschland und in den Vereinigten Staaten, Meisenheim am Glan, Anton Hain.

Infratest Sozialforschung (1989), Sonderauswertung Republikaner: Kumulierte Ergebnisse von vier Reprasentativuntersuchungen, in Sozialstruktur und Einstellungen von Wablern rechtsextremer Parteien, Bonn, Vorwärts Verlag, pp. 23-87.

Jaschke, H.G. (1989), Verschlungene Traditionen: Zur geschichte des Rechtsextremismus in der Bundesrepublik, in «Gewerkschaftliche Monatshefte», XL, n. 9, pp. 513-523.

Kaltefleiter, W. (1968), Wirtschaft und Politik in Deutschland: Konjunktur als Bestimmungsfaktor des Parteiensystems, Köln e Opladen, Westdeutscher Verlag.

Klingemann, H.D. e F.U. Pappi (1972), Politischer Radikalismus: Theoretische und methodische Probleme der Radikalismusforschung, München e Wien, Oldenbourg.

Kornhauser, W. (1960), The Politics of Mass Society, London, Routledge and Kegan Paul.

Lepszy, N. (1989), Die Republikaner: Ideologie Program Organisation, in «Politik und Zeitgeschichte», n. 41-42, pp. 8-9.

Liepelt, K. (1967), Anbänger der neuen Rechtspartei: Ein Beitrag zur Diskussion über das Wählerreservoir der NPD, in «Politische Vierteljahresschrift», VIII, pp. 237-71.

Lipset, S.M. (1960), Political Man: The Social Bases of Politics, Garden City (N.Y.), Doubleday.

May, D. (1992), The Bremen Land Election of September 1991, in «German Politics», I, n. 1, pp. 119-123.

Neubauer, H. (1985), Wo bleibt die deutsche Rechte?, in «Deutsche Annalen. Jahrbuch des Nationalgeschehens», Leoni, Druffel, pp. 134-156.

Niedermayer, O. (1990), Sozialstruktur, politische Orientierungen und die Unterstützung extrem rechter Parteien in Westeuropa, in «Zeitschrift für Parlamentsfragen», XXI, n. 4, pp. 564-82.

Noelle-Neumann, E. (1986), Spirial of Silence: Our Social Skin, Chicago, University of Chicago Press.

Pappi, F.U. (1990), Die Republikaner im Parteienysystem der Bundesrepublik. Protesterscheinung oder politische Alternative?, in «Politik und Zeitgeschichte», n. 21, pp. 37-44.

Parkes, C. (1993), Estreme right makes sweeping gains in German election, in «Financial Times», 8 marzo, p. 16.

Paul, G. (1989), Der Schatten verblaßt: Die Normalisierung des Rechtsextremismus in den achtziger Jabren, in G. Paul (a cura di), Hitlers Schatten verblaßt: Die Normalisierung des Rechtsextremismus, Bonn, J.H.W. Dietz, pp. 11-48.

Roberts, G.K. (1992), Right-Wing Radicalism in the New Germany, in «Parliamentary Affairs», VL, n. 3, pp. 327-344. 
Roth, D. (1989), Sind die Republikaner die fünfte Partei? Sozial und Meinungsstrukturen der Wähler der Republikaner, in «Politik und Zeitgeschichte», n. 4, pp. 10-20.

Roth, D. (1990a), Republikaner: Schneller Aufstieg und tiefer Fall einer Protestpartei am rechten Rand, in «Politik und Zeitgeschichte», n. 37-38, pp. 27-39.

- (1990b), Sind die Republikaner die fünfte Partei? Sozial und Meinungsstrukturen der Wäbler der Republikaner, paper presentato alla Conferenza Internazionale su «Rechtsextremismus im westeruropäischen Kontext», Antwerp.

Rowold, M. (1974), Im Schatten der Macht: Zur Oppositionsrolle der nicht etablierten Parteien in der Bundesrepublik, Düsseldorf, Droste.

Saalfeld, T. (1993), The Politics of National-Populism: Ideology and Policies of the German Republikaner Party, in «German Politics», II, n. 2, pp. 177-199.

Scheuch, E.K. (1974), Politischer Extremismus in der Bundesrepublik, in Löwenthal e H.P. Schwartz (a cura di), Die zweite Republik. 25 Jabre Bundesrepublik Deutschland: eine Bilanz, Stuttgart, Seewald, pp. 433-69.

- (1990), Die Suche nach der Besonderbeit der beutigen Deutschen, in «Kölner Zeitschrift für Soziologie und Sozialpsychologie», XLII, pp. 734-52.

Scheuch, E.K. e H.D. Klingemann (1967), Theorie des Rechtsradikalismus in westlichen Industriegesellschaften, in «Hamburger Jahrbuch für Wirtschafts und Gesellschaftpolitik», XII, pp. 11-29.

Schmidt, G. (1968), Ideologie und Propaganda der NPD, in «Politik und Zeitgeschichte», n. 7, pp. 3-22.

Schmollinger, H.W. (1986a), Die Deutsche Konservative Partei - Deutsche Rechtspartei, in R. Stöss (a cura di), Parteienbandbuch: Die Parteien der Bundesrepublik Deutschland 1945-1980, II volume, Opladen, Westdeutscher Verlag, pp. 982-1024.

- (1986b), Die Deutsche Reichspartei, in R. Stöss (a cura di), Parteienbandbuch: Die Parteien der Bundesrepublik Deutschland 19451980, II volume, Oplanden, Westdeutscher Verlag, pp. 11121191.

- (1986c), Die Nationaldemokratische Partei Deutschlands, in R. Stöss (a cura di), Parteienhandbuch: Die Parteien der Bundesrepublik Deutschland 1945-1980, IV volume, Opladen, Westdeutscher Verlag, pp. 1922-1994.

- (1986d), Die Sozialistische Reichspartei, in R. Stöss (a cura di), Parteienbandbuch: Die Parteien der Bundesrepublik Deutschland 1945-1980, IV volume, Opladen, Westdeutscher Verlag, pp. 2274-2336.

Sinus Gesellschaft für Sozialforschung und Marktforschung (1981), 5 Million Deutsche: «Wir sollten wieder einen Fübrer haben...»: Die 
SINUS-Studie über rechtsextremistische Einstellungen bei den Deutschen, Reinbek, Rowohlt.

«Der Spiegel» (1989), Fünfte Partei in den Bundestag?, 27 febbraio, pp. 44-53.

Statistisches Bundesamt (a cura di) (1989), 40 Jabre Wablen in der Bundesrepublik Deutschland, Stuttgart, Metzler-Poeschel.

Stöss, R. (1986a), Die Aktionsgemeinschaft Unabbängiger Deutscher, in R. Stöss (a cura di), Parteienbandbuch: Die Parteien der Bundesrepublik Deutschland 1945-1980, I volume, Opladen, Westdeutscher Verlag, pp. 310-335.

- (1986b), Die Deutsche Gemeinschaft, in R. Stöss (a cura di), Parteienhandbuch: Die Parteien der Bundesrepublik Deutschland 1945. 1980, II volume, Opladen, Westdeutscher Verlag, pp. 877-900.

- (1986c), Einleitung: Struktur und Entwicklung des Parteiensystems der Bundesrepublik - Eine Theorie, in R. Stöss (a cura di), Parteienhandbuch: Die Parteien der Bundesrepublik Deutschland 1945. 1980, I volume, Opladen, WestdeutscherVerlag, pp. 17-309.

- (1989), Die extreme Rechte in der Bundesrepublik: Entwicklung Ursachen - Gegemmaßnabmen, Opladen, Westdeutscher Verlag.

- (1990), Die «Republikaner»: Wober sie kommen. Was sie wollen. Wer sie wählt. Was zu tun ist, Köln, Bund-Verlag.

Tauber, P. (1967), Beyond Eagle and Swastika: German Nationalism since 1945, 2 volumi, Middletown, Wesleyan University Press.

Veen, H.-J., N. Lepszy e P. Mnich (1991), Die Republikaner-Partei zu Beginn der 90er Jabre - Programm, Propaganda, Organisation, Wähler- und Sympatbisantestruktur, Sankt Augustin, Forschngsinstitut der Konrad-Adenauer-Stiftung, Interne Studien, n. 14.

Winker, H.A. (1972), Demokratie und Nationalsozialismus: Die politische Entwicklung von Handwerk und Kleinbandel in der Weimarer Republik, Köln, Kiepenheuer und Witsch.

Zimmermann, E. e T. Saalfeld (1994), Right-Wing Extremism in Germany. From 1949 to the Present: Towards a Supply-Side Model, non pubblicato. 